\title{
A Better Algorithm for Random $k$-SAT
}

\author{
Amin Coja-Oghlan* \\ University of Edinburgh, School of Informatics, Edinburgh EH8 9AB, UK \\ acoghlaneinf.ed.ac.uk
}

October 23, 2018

\begin{abstract}
Let $\Phi$ be a uniformly distributed random $k$-SAT formula with $n$ variables and $m$ clauses. We present a polynomial time algorithm that finds a satisfying assignment of $\boldsymbol{\Phi}$ with high probability for constraint densities $m / n<\left(1-\varepsilon_{k}\right) 2^{k} \ln (k) / k$, where $\varepsilon_{k} \rightarrow 0$. Previously no efficient algorithm was known to find solutions with non-vanishing probability beyond $m / n=1.817 \cdot 2^{k} / k$ [Frieze and Suen, J. of Algorithms 1996].

Key words: random structures, efficient algorithms, phase transitions, $k$-SAT.
\end{abstract}

\section{Introduction}

\subsection{Solving random $k$-SAT}

The $k$-SAT problem is well known to be NP-hard for $k \geq 3$, and in fact no algorithm with a sub-exponential worst-case running time is known to decide whether a given $k$-SAT formula has a satisfying assignment. Nevertheless, that $k$-SAT is NP-hard merely indicates that no algorithm can solve all possible inputs efficiently. Therefore, there has been a significant amount of research on heuristics for $k$-SAT, i.e., algorithms that solve "most" inputs efficiently, where the meaning of "most" depends on the scope of the respective paper. While some heuristics for $k$-SAT are very sophisticated, virtually all of them are based on (at least) one of the following basic paradigms.

Pure literal rule. If a variable $x$ occurs only positively (resp. negatively) in the formula, set it to true (resp. false). Simplify the formula by substituting the newly assigned value for $x$ and repeat.

Unit clause propagation. If the formula contains a clause that contains only a single literal ("unit clause"), then set the underlying variable so as to satisfy this clause. Then simplify the formula and repeat.

Walksat. Initially pick a random assignment. Then repeat the following. While there is an unsatisfied clause, pick one at random, pick a variable occurring in the chosen clause randomly, and flip its value.

Backtracking. Assign a variable $x$, simplify the formula, and recurse. If the recursion fails to find a satisfying assignment, assign $x$ the opposite value and recurse.

Heuristics based on these paradigms can be surprisingly successful (given that $k$-SAT is NP-hard) on certain types of inputs (e.g., [10, 16]). However, it remains remarkably simple to generate formulas that elude all known algorithms/heuristics. Indeed, the simplest conceivable type of random instances does the trick: let $\boldsymbol{\Phi}$ denote a $k$-SAT formula over the variable set $V=\left\{x_{1}, \ldots, x_{n}\right\}$ that is obtained by choosing $m$ clauses uniformly at random and independently from the set of all $(2 n)^{k}$ possible clauses. Then for a large regime of constraint densities $m / n$ satisfying assignments are known to exist due to non-constructive arguments, but no algorithm is known to find one in sub-exponential time with a non-vanishing probability.

*S Supported by EPSRC grant EP/G039070/1. 
To be precise, keeping $k$ fixed and letting $m=\lceil r n\rceil$ for a fixed $r>0$, we say that $\boldsymbol{\Phi}$ has some property with high probability ("w.h.p.") if the probability that the property holds tends to one as $n \rightarrow \infty$. Via the (highly non-algorithmic) second moment method and the sharp threshold theorem [3, 4, 14] it can be shown that $\boldsymbol{\Phi}$ has a satisfying assignment w.h.p. if $m / n<\left(1-\varepsilon_{k}\right) 2^{k} \ln 2$. Here $\varepsilon_{k}$ tends to 0 for large $k$. On the other hand, a first moment argument shows that no satisfying assignment exists w.h.p. if $m / n>2^{k} \ln 2$. In summary, the threshold for $\boldsymbol{\Phi}$ being satisfiable is asymptotically $2^{k} \ln 2$.

But for densities $m / n$ beyond $O\left(2^{k} / k\right)$ no algorithm has been known to find a satisfying assignment in polynomial time with probability $\Omega(1)$ - neither on the basis of a rigorous analysis, nor on the basis of experimental or other evidence. In fact, many algorithms, including Pure Literal, Unit Clause, and DPLL-type algorithms, are known to fail or exhibit an exponential running time beyond $O\left(2^{k} / k\right)$. There is experimental evidence that the same is true of Walksat. Indeed, devising an algorithm to solve random formulas with a non-vanishing probability for densities $m / n$ up to $2^{k} \omega(k) / k$ for any $\omega(k) \rightarrow \infty$ has been a well-known open problem [3, 4, 8, 21], which the following theorem resolves.

Theorem 1.1 There are a sequence $\varepsilon_{k} \rightarrow 0$ and a polynomial time algorithm $F$ ix such that $F i x$ applied to a random formula $\boldsymbol{\Phi}$ with $m / n \leq\left(1-\varepsilon_{k}\right) 2^{k} \ln (k) / k$ outputs a satisfying assignment w.h.p.

Fix is a combinatorial, local-search type algorithm. It can be implemented to run in time $O(n+m)^{3 / 2}$.

The recent paper [2] provides evidence that beyond density $m / n=2^{k} \ln (k) / k$ the problem of finding a satisfying assignment becomes conceptually significantly more difficult (to say the least). To explain this, we need to discuss a concept that originates from statistical physics.

\subsection{A digression: replica symmetry breaking}

For the last decade random $k$-SAT has been studied by statistical physicists using sophisticated, insightful, but mathematically highly non-rigorous techniques from the theory of spin glasses. Their results suggest that below the threshold density $2^{k} \ln 2$ for the existence of satisfying assignments various other phase transitions take place that affect the performance of algorithms.

To us the most important one is the dynamic replica symmetry breaking (dRSB) transition. Let $S(\boldsymbol{\Phi}) \subset$ $\{0,1\}^{V}$ be the set of all satisfying assignments of the random formula $\boldsymbol{\Phi}$. We turn $S(\boldsymbol{\Phi})$ into a graph by considering $\sigma, \tau \in S(\boldsymbol{\Phi})$ adjacent if their Hamming distance equals one. Very roughly speaking, according to the dRSB hypothesis there is a density $r_{R S B}$ such that for $m / n<r_{R S B}$ the correlations that shape the set $S(\boldsymbol{\Phi})$ are purely local, whereas for densities $m / n>r_{R S B}$ long range correlations occur. Furthermore, $r_{R S B} \sim 2^{k} \ln (k) / k$.

Confirming and elaborating on this hypothesis, we recently established a good part of the dRSB phenomenon rigorously [2]. In particular, we proved that there is a sequence $\varepsilon_{k} \rightarrow 0$ such that for $m / n>\left(1+\varepsilon_{k}\right) 2^{k} \ln (k) / k$ the values that the solutions $\sigma \in S(\boldsymbol{\Phi})$ assign to the variables are mutually heavily correlated in the following sense. Let us call a variable $x$ frozen in a satisfying assignment $\sigma$ if any satisfying assignment $\tau$ such that $\sigma(x) \neq \tau(x)$ is at Hamming distance $\Omega(n)$ from $\sigma$. Then for $m / n>\left(1+\varepsilon_{k}\right) 2^{k} \ln (k) / k$ in all but a $o(1)$-fraction of all solutions $\sigma \in S(\boldsymbol{\Phi})$ all but an $\varepsilon_{k}$-fraction of the variables are frozen w.h.p., where $\varepsilon_{k} \rightarrow 0$.

This suggests that on random formulas with density $m / n>\left(1+\varepsilon_{k}\right) 2^{k} \ln (k) / k$ local search algorithms are unlikely to succeed. For think of the factor graph, whose vertices are the variables and the clauses, and where a variable is adjacent to all clauses in which it occurs. Then a local search algorithm assigns a value to a variable $x$ on the basis of the values of the variables that have distance $O(1)$ from $x$ in the factor graph. But in the random formula $\boldsymbol{\Phi}$ with $m / n>\left(1+\varepsilon_{k}\right) 2^{k} \ln (k) / k$ assigning one variable $x$ is likely to impose constraints on the values that can be assigned to variables at distance $\Omega(\ln n)$ from $x$ in the factor graph.

The above discussion applies to "large" values of $k$ (say, $k \geq 10$ ). In fact, non-rigorous arguments as well as experimental evidence [5] suggest that the picture is quite different and rather more complicated for "small" $k$ (say, $k=3,4,5$ ). In this case the various phenomena that occur at (or very near) the point $2^{k} \ln (k) / k$ for $k \geq 10$ appear to happen at vastly different points in the satisfiable regime. To keep matters as simple as possible we focus on "large" $k$ in this paper. In particular, no attempt has been made to derive explicit bounds on the numbers $\varepsilon_{k}$ in Theorem 1.1 for "small" values of $k$. 


\begin{tabular}{|c|c|c|c|}
\hline Algorithm & Density $m / n<\cdots$ & Success probability & Ref., year \\
\hline Pure Literal (“PL") & $o(1)$ as $k \rightarrow \infty$ & w.h.p. & [19], 2006 \\
\hline $\begin{array}{c}\text { Walksat, rigorous } \\
\text { Walksat, non-rigorous }\end{array}$ & $\begin{array}{c}\frac{1}{6} \cdot 2^{k} / k^{2} \\
2^{k} / k\end{array}$ & $\begin{array}{l}\text { w.h.p. } \\
\text { w.h.p. }\end{array}$ & $\begin{array}{l}{[9], 2009} \\
{[22], 2003}\end{array}$ \\
\hline Unit Clause (“UC”) & $\frac{1}{2}\left(\frac{k-1}{k-2}\right)^{k-2} \cdot \frac{2^{k}}{k}$ & $\Omega(1)$ & [7], 1990 \\
\hline Shortest Clause (“SC”) & $\frac{1}{8}\left(\frac{k-1}{k-3}\right)^{k-3} \frac{k-1}{k-2} \cdot \frac{2^{k}}{k}$ & w.h.p. & [8], 1992 \\
\hline SC+backtracking ("SCB") & $\sim 1.817 \cdot \frac{2^{k}}{k}$ & w.h.p. & [15], 1996 \\
\hline $\begin{array}{c}\mathrm{BP}+\text { decimation ("BPdec") } \\
\text { (non-rigorous) }\end{array}$ & $\mathrm{e} \cdot 2^{k} / k$ & w.h.p. & [21], 2007 \\
\hline
\end{tabular}

Table 1: Algorithms for random $k$-SAT

\subsection{Related work}

Quite a few papers deal with efficient algorithms for random $k$-SAT, contributing either rigorous results, non-rigorous evidence based on physics arguments, or experimental evidence. Table 1 summarizes the part of this work that is most relevant to us. The best rigorous result (prior to this work) is due to Frieze and Suen [15], who proved that "SCB" succeeds for densities $\eta_{k} 2^{k} / k$, where $\eta_{k}$ is increasing to 1.817 as $k \rightarrow \infty$. SCB can be considered a (restricted) DPLL-algorithm. More precisely, SCB combines the shortest clause rule, which is a generalization of Unit Clause, with (very limited) backtracking. Conversely, it is known that DPLL-type algorithms require an exponential running time w.h.p. for densities beyond $O\left(2^{k} / k\right)$ [1].

Montanari, Ricci-Tersenghi, and Semerjian [21] provide evidence that Belief Propagation guided decimation may succeed up to density $\mathrm{e} \cdot 2^{k} / k$. This algorithm is based on a very different paradigm than the others mentioned in Table 1 The basic idea is to run a message passing algorithm ("Belief Propagation") to compute for each variable the marginal probability that this variable takes the value true/false in a uniformly random satisfying assignment. Then, the decimation step selects a variable, assigns it the value true/false with the corresponding marginal probability, and simplifies the formula. Ideally, repeating this procedure will yield a satisfying assignment, provided that Belief Propagation keeps yielding the correct marginals. Proving (or disproving) this remains a major open problem.

Survey Propagation is a modification of Belief Propagation that aims to approximate the marginal probabilities induced by a particular (non-uniform) probability distribution on the set of satisfying assignments [6]. It can be combined with a decimation procedure as well to obtain a heuristic for finding a satisfying assignment. There is (non-rigorous) evidence that for most of the satisfiable regime (actually $\left.m / n<2^{k} \ln 2-O(1)\right)$ Belief and Survey Propagation are essentially equivalent [20]. Hence, there is no evidence that Survey Propagation finds satisfying assignments beyond $O\left(2^{k} / k\right)$ for general $k$.

In summary, various algorithms are known/appear to succeed for densities $c \cdot 2^{k} / k$, where the constant $c$ depends on the particulars of the algorithm. But I am not aware of prior evidence (either rigorous results, non-rigorous arguments, or experiments) that some algorithm succeeds for densities $m / n=2^{k} \omega(k) / k$ with $\omega(k) \rightarrow \infty$.

The discussion so far concerns the case of general $k$. In addition, a large number of papers deal with the case $k=3$. Flaxman [13] provides a survey. Currently the best rigorously analyzed algorithm for random 3-SAT is known to succeed up to $m / n=3.52$ [17]. This is also the best known lower bound on the 3-SAT threshold. The best current upper bound is 4.506 [11], and non-rigorous arguments suggest the threshold to be $\approx 4.267$ [6]. As mentioned in Section [1.2, there is non-rigorous evidence that the structure of the set of all satisfying assignment evolves differently in random 3-SAT than in random $k$-SAT for "large" $k$. This may be why experiments suggest that Survey Propagation guided decimation for 3-SAT succeeds for densities $m / n$ up to 4.2 [6].

\subsection{Techniques and outline}

The algorithms for random $k$-SAT from [7, 8, 15] all follow a very simple scheme: 
Initially all variables are unassigned. In each step apply some rule (referring to the previously assigned variables/values only) to select a currently unassigned variable. Assign the selected variable for good, simplify the formula, and proceed.

The Unit Clause algorithm is the prototypical example: the underlying rule is to check if there is a clause that has $k-1$ false literals due previous decisions; if so, the algorithm sets the last unassigned variable so as to satisfy the clause. Otherwise the algorithm picks an unassigned variable randomly and assigns it a random value. (The algorithm SCB from [15] deviates from this pattern slightly as it may backtrack to revise previous assignments, but this happens at most $O\left(\ln ^{2} n\right)$ times w.h.p.)

The analysis of such algorithms is based on the "method of deferred decisions". Suppose we apply the algorithm to a random formula and condition on the occurrences of the variables assigned in the first $t$ steps. Assume that these are precisely the variables $x_{1}, \ldots, x_{t}$. Then all the literals whose underlying variable is none of $x_{1}, \ldots, x_{t}$ remain stochastically independent and uniformly distributed over set of the remaining $2(n-t)$ literals. This fact makes it possible to either model the execution of the algorithm by differential equations [7, 8], or by a Markov chain [15]. Of course, this type of analysis crucially exploits the fact that the algorithm (almost) never revises previous decisions.

Instead of assigning one variable at a time, the Walksat algorithm starts from a complete (e.g., randomly chosen) assignment of truth values to all the variables. Of course, this initial assignment is very unlikely to be satisfying. Hence, while there is an unsatisfied clause, the algorithm picks one of them at random and flips the value of a randomly chosen variable occurring in that clause. Since Walksat actually starts from a complete assignment and may flip the value of the same variable several times, the method of deferred decisions does not apply. In fact, although experimental (and non-rigorous) evidence suggests that Walksat finds a satisfying assignment in linear time w.h.p. for $m / n<2^{k} / k$, the best current rigorous analysis only shows this for $m / n<2^{k} /\left(6 k^{2}\right)$ [9]. (The proof is based on relating Walksat to a branching process.)

The algorithm Fix for Theorem 1.1 is similar to Walksat in that it starts with a complete assignment say, for the sake of concreteness, the one that sets all variables to true. The number of unsatisfied clauses is $(1+o(1)) 2^{-k} m$ w.h.p. To reach a satisfying assignment, $\mathrm{Fix}$ will have to flip (at least) one variable from each of these clauses. But in contrast to Walksat, Fix does not choose this variable randomly. Instead Fix applies a greedy rule: whenever possible choose a variable $x$ so that flipping $x$ does not generate new unsatisfied clauses. Thus, one could consider Fix a greedy version of Walksat. We will describe the algorithm precisely in Section 3

The analysis of $\mathrm{Fix}$ is based on a blend of probabilistic methods (e.g., martingales) and combinatorial arguments. We can employ the method of deferred decisions to a certain extent: the analysis "pretends" that the algorithm exposes the literal occurrences of the random input formula only when it becomes strictly necessary, so that the unexposed ones remain "random". However, the picture is not as clean as in the analysis of, say, Unit Clause. The reason is that we will have to track certain rather non-trivial random variables throughout the process, for which we will resort to a direct combinatorial analysis. Section 3 contains an outline of the analysis, the details of which are carried out in Section 46 Before we come to this, we need a few preliminaries.

\section{Preliminaries and notation}

In this section we introduce some notation and present a few basic facts. Although most of them (or closely related ones) are well known, we present some of the proofs for the sake of completeness.

\subsection{Balls and bins}

Consider a balls and bins experiment where $\mu$ distinguishable balls are thrown independently and uniformly at random into $n$ bins. Thus, the probability of each distribution of balls into bins equals $n^{-\mu}$.

Lemma 2.1 Let $\mathcal{Z}(\mu, n)$ be the number of empty bins. Let $\lambda=n \exp (-\mu / n)$. Then $\mathrm{P}[\mathcal{Z}(\mu, n) \leq \lambda / 2] \leq$ $O(\sqrt{\mu}) \cdot \exp (-\lambda / 8)$ as $n \rightarrow \infty$. 
The proof is based on the following Chernoff bound on the tails of a binomially distributed random variable $X$ with mean $\lambda$ (see [18, pages 26-28]): for any $t>0$

$$
\mathrm{P}(X \geq \lambda+t) \leq \exp \left(-\frac{t^{2}}{2(\lambda+t / 3)}\right) \quad \text { and } \quad \mathrm{P}(X \leq \lambda-t) \leq \exp \left(-\frac{t^{2}}{2 \lambda}\right)
$$

Proof of Lemma 2.1] Let $X_{i}$ be the number of balls in bin $i$. In addition, let $\left(Y_{i}\right)_{1 \leq i \leq n}$ be a family of mutually independent Poisson variables with mean $\mu / n$, and let $Y=\sum_{i=1}^{n} Y_{i}$. Then $Y$ has a Poisson distribution with mean $\mu$. Therefore, Stirling's formula shows $\mathrm{P}[Y=\mu]=\Theta\left(\mu^{-1 / 2}\right)$. Furthermore, the conditional joint distribution of $Y_{1}, \ldots, Y_{n}$ given that $Y=\mu$ coincides with the joint distribution of $X_{1}, \ldots, X_{n}$ (see, e.g., [12, Section 2.6]). As a consequence,

$$
\begin{aligned}
\mathrm{P}[\mathcal{Z}(\mu, n) \leq \lambda / 2] & =\mathrm{P}\left[\left|\left\{i \in[n]: Y_{i}=0\right\}\right|<\lambda / 2 \mid Y=\mu\right] \\
& \leq \frac{\mathrm{P}\left[\left|\left\{i \in[n]: Y_{i}=0\right\}\right|<\lambda / 2\right]}{\mathrm{P}[Y=\mu]}=O(\sqrt{\mu}) \cdot \mathrm{P}\left[\left|\left\{i \in[n]: Y_{i}=0\right\}\right|<\lambda / 2\right.
\end{aligned}
$$

Finally, since $Y_{1}, \ldots, Y_{n}$ are mutually independent and $\mathrm{P}\left[Y_{i}=0\right]=\lambda / n$ for all $1 \leq i \leq n$, the number of indices $i \in[n]$ such that $Y_{i}=0$ is binomially distributed with mean $\lambda$. Thus, the assertion follows from (2) and the Chernoff bound (1).

\subsection{Random $k$-SAT formulas}

Throughout the paper we let $V=V_{n}=\left\{x_{1}, \ldots, x_{n}\right\}$ be a set of propositional variables. If $Z \subset V$, then $\bar{Z}=\{\bar{x}: x \in Z\}$ contains the corresponding set of negative literals. Moreover, if $l$ is a literal, then $|l|$ signifies the underlying propositional variable. If $\mu$ is an integer, let $[\mu]=\{1,2, \ldots, \mu\}$.

We let $\Omega_{k}(n, m)$ be the set of all $k$-SAT formulas with variables from $V=\left\{x_{1}, \ldots, x_{n}\right\}$ that contain precisely $m$ clauses. More precisely, we consider the formula an ordered $m$-tuple of clauses and each clause an ordered $k$-tuples of literals, allowing both literals to occur repeatedly in one clause and clauses to occur repeatedly in the formula. Let $\Sigma_{k}(n, m)$ be the power set of $\Omega_{k}(n, m)$, and let $\mathrm{P}=\mathrm{P}_{k}(n, m)$ be the uniform probability measure (which assigns probability $(2 n)^{-k m}$ to each formula). We obtain a probability space $\left(\Omega_{k}(n, m), \Sigma_{k}(n, m), \mathrm{P}\right)$.

Throughout the paper we denote a random element of $\Omega_{k}(n, m)$ by $\boldsymbol{\Phi}$. Unless otherwise specified, $\boldsymbol{\Phi}$ is uniformly distributed. In addition, we use $\Phi$ to denote specific (i.e., non-random) elements of $\Omega_{k}(n, m)$. If $\Phi \in \Omega_{k}(n, m)$, then $\Phi_{i}$ denotes the $i$ th clause of $\Phi$, and $\Phi_{i j}$ denotes the $j$ th literal of $\Phi_{i}$.

Lemma 2.2 For any $\delta>0$ and any $k \geq 3$ there is $n_{0}>0$ such that for all $n>n_{0}$ the following is true. Suppose that $m \geq \delta n$ and that $X_{i}: \Omega_{k}(n, m) \rightarrow\{0,1\}$ is a random variable for each $i \in[m]$. Let $\mu=\left\lceil\ln ^{2} n\right\rceil$. If there is a number $\lambda \geq \delta$ such that for any set $M \subset[m]$ of size $\mu$ we have

$$
\mathrm{E}\left[\prod_{i \in M} X_{i}\right] \leq \lambda^{\mu}, \text { then } \mathrm{P}\left[\sum_{i=1}^{m} X_{i} \geq(1+\delta) \lambda m\right]<n^{-10} .
$$

Proof. Let $\mathcal{M}$ be the number of sets $M \subset[m]$ of size $\mu$ such that $\prod_{i \in M} X_{i}=1$. Then $\mathrm{E}[\mathcal{M}] \leq\left(\begin{array}{c}m \\ \mu\end{array}\right) \lambda^{\mu}$. If $X=\sum_{i=1}^{m} X_{i} \geq L=\lceil(1+\delta) \lambda m\rceil$, then $\mathcal{M} \geq\left(\begin{array}{l}L \\ \mu\end{array}\right)$. Consequently, by Markov's inequality

$$
\mathrm{P}[X \geq L] \leq \mathrm{P}\left[\mathcal{M} \geq\left(\begin{array}{c}
L \\
\mu
\end{array}\right)\right] \leq \frac{\mathrm{E}[\mathcal{M}]}{\left(\begin{array}{l}
L \\
\mu
\end{array}\right)} \leq \frac{\left(\begin{array}{l}
m \\
\mu
\end{array}\right) \lambda^{\mu}}{\left(\begin{array}{l}
L \\
\mu
\end{array}\right)} \leq\left(\frac{\lambda m}{L-\mu}\right)^{\mu} \leq\left(\frac{\lambda m}{(1+\delta) \lambda m-\mu}\right)^{\mu}
$$

Since $\lambda m \geq \delta^{2} n$ we see that $(1+\delta) \lambda m-\mu \geq(1+\delta / 2) \lambda m$ for sufficiently large $n$. Hence, $\mathrm{P}[X \geq L] \leq$ $(1+\delta / 2)^{-\mu}<n^{-10}$ for large enough $n$.

Although we allow variables to appear repeatedly in the same clause, the following lemma shows that this occurs very rarely w.h.p. 
Lemma 2.3 Suppose that $m=O(n)$. Then w.h.p. there are at most $\ln n$ indices $i \in[m]$ such that one of the following is true.

1. There are $1 \leq j_{1}<j_{2} \leq k$ such that $\left|\mathbf{\Phi}_{i j_{1}}\right|=\left|\boldsymbol{\Phi}_{i j_{2}}\right|$.

2. There is $i^{\prime} \neq i$ and indices $j_{1} \neq j_{2}, j_{1}^{\prime} \neq j_{2}^{\prime}$ such that $\left|\mathbf{\Phi}_{i j_{1}}\right|=\left|\boldsymbol{\Phi}_{i^{\prime} j_{1}^{\prime}}\right|$ and $\left|\mathbf{\Phi}_{i j_{2}}\right|=\left|\boldsymbol{\Phi}_{i^{\prime} j_{2}^{\prime}}\right|$.

Furthermore, w.h.p. no variable occurs in more than $\ln ^{2} n$ clauses.

Proof. Let $X$ be the number of such indices $i$ for which 1 . holds. For each $i \in[m]$ and any pair $1 \leq j_{1}<$ $j_{2} \leq k$ the probability that $\left|\boldsymbol{\Phi}_{i j_{1}}\right|=\left|\boldsymbol{\Phi}_{i j_{2}}\right|$ is $1 / n$, because each of the two variables is chosen uniformly at random. Hence, by the union bound the probability that there are $j_{1}, j_{2}$ such that $\left|\boldsymbol{\Phi}_{i j_{1}}\right|=\left|\boldsymbol{\Phi}_{i j_{2}}\right|$ is at most $\left(\begin{array}{l}k \\ 2\end{array}\right) / n$. Consequently, $\mathrm{E}[X] \leq m\left(\begin{array}{c}k \\ 2\end{array}\right) / n=O(1)$, and thus $X \leq \frac{1}{2} \ln n$ w.h.p. by Markov's inequality.

Let $Y$ be the number of $i \in[m]$ for which 2 . is true. For any given $i, i^{\prime}, j_{1}, j_{1}^{\prime}, j_{2}, j_{2}^{\prime}$ the probability that $\left|\boldsymbol{\Phi}_{i j_{1}}\right|=\left|\boldsymbol{\Phi}_{i^{\prime} j_{1}^{\prime}}\right|$ and $\left|\boldsymbol{\Phi}_{i j_{2}}\right|=\left|\boldsymbol{\Phi}_{i^{\prime} j_{2}^{\prime}}\right|$ is $1 / n^{2}$. Furthermore, there are $m^{2}$ ways to choose $i, i^{\prime}$ and then $(k(k-1))^{2}$ ways to choose $j_{1}, j_{1}^{\prime}, j_{2}, j_{2}^{\prime}$. Hence, $\mathrm{E}[Y] \leq m^{2} k^{4} n^{-2}=O(1)$. Thus, $X \leq \frac{1}{2} \ln n$ w.h.p. by Markov's inequality.

Finally, for any variable $x$ the number of indices $i \in[m]$ such that $x$ occurs in $\boldsymbol{\Phi}_{i}$ has a binomial distribution $\operatorname{Bin}\left(m, 1-(1-1 / n)^{k}\right)$. Since the mean $m \cdot\left(1-(1-1 / n)^{k}\right)$ is $O(1)$, the Chernoff bound (1) implies that the probability that $x$ occurs in more than $\ln ^{2} n$ clauses is $o(1 / n)$. Hence, by the union bound there is no variable with this property w.h.p.

Recall that a filtration is a sequence $\left(\mathcal{F}_{t}\right)_{0 \leq t \leq \tau}$ of $\sigma$-algebras $\mathcal{F}_{t} \subset \Sigma_{k}(n, m)$ such that $\mathcal{F}_{t} \subset \mathcal{F}_{t+1}$ for all $0 \leq t<\tau$. For a random variable $X$ we let $\mathrm{E}\left[X \mid \mathcal{F}_{t}\right]$ denote the conditional expectation (which is a random variable). Remember that $\mathrm{P}\left[\cdot \mid \mathcal{F}_{t}\right]$ assigns a probability measure $\mathrm{P}\left[\cdot \mid \mathcal{F}_{t}\right](\Phi)$ to any $\Phi \in \Omega_{k}(n, m)$, namely

$$
\mathrm{P}\left[\cdot \mid \mathcal{F}_{t}\right](\Phi): A \in \Sigma_{k}(n, m) \mapsto \mathrm{E}\left[\mathbf{1}_{A} \mid \mathcal{F}_{t}\right](\Phi),
$$

where $\mathbf{1}_{A}(\varphi)=1$ if $\varphi \in A$ and $\mathbf{1}_{A}(\varphi)=0$ otherwise.

Lemma 2.4 Let $\left(\mathcal{F}_{t}\right)_{0 \leq t \leq \tau}$ be a filtration and let $\left(X_{t}\right)_{1 \leq t \leq \tau}$ be a sequence of random variables such that each $X_{t}$ is $\mathcal{F}_{t}$-measurable. Assume that there are numbers $\xi_{t} \geq 0$ such that $\mathrm{E}\left[X_{t} \mid \mathcal{F}_{t-1}\right] \leq \xi_{t}$ for all $t$. Then $\mathrm{E}\left[\prod_{1 \leq t \leq \tau} X_{t} \mid \mathcal{F}_{0}\right] \leq \prod_{1 \leq t \leq \tau} \xi_{t}$.

Proof. For $1 \leq s \leq \tau$ we let $Y_{s}=\prod_{t=1}^{s} X_{t}$. Let $s>1$. Since $Y_{s-1}$ is $\mathcal{F}_{s-1}$-measurable, we obtain $\mathrm{E}\left[Y_{s} \mid \mathcal{F}_{0}\right]=\mathrm{E}\left[Y_{s-1} X_{s} \mid \mathcal{F}_{0}\right]=\mathrm{E}\left[\mathrm{E}\left[Y_{s-1} X_{s} \mid \mathcal{F}_{s-1}\right] \mid \mathcal{F}_{0}\right]=\mathrm{E}\left[Y_{s-1} \mathrm{E}\left[X_{s} \mid \mathcal{F}_{s-1}\right] \mid \mathcal{F}_{0}\right] \leq \xi_{s} \mathrm{E}\left[Y_{s-1} \mid \mathcal{F}_{0}\right]$, whence the assertion follows by induction. $\square$ We also need the following tail bound ("Azuma-Hoeffding", e.g. [18, p. 37]).

Lemma 2.5 Let $\left(M_{t}\right)_{0 \leq t \leq \tau}$ be a martingale such that $M_{0}=\mathrm{E}\left[M_{\tau}\right]$. Suppose that $\left|M_{t}-M_{t-1}\right| \leq c_{t}$ for all $1 \leq t \leq \tau$. Then for any $\lambda>0 \mathrm{P}\left[\left|M_{\tau}-M_{0}\right|>\lambda\right] \leq \exp \left[-\lambda^{2} /\left(2 \sum_{t=1}^{\tau} c_{t}^{2}\right)\right]$.

Finally, we need the following bound on the number of clauses that have "few" positive literals in total but contain at least one positive variable from a "small" set.

Lemma 2.6 There is a constant $\alpha>0$ such that for all $k \geq 3$ and $m / n \leq 2^{k} k^{-1} \ln k$ the following is true. Let $1 \leq l \leq \sqrt{k}$ and set $\delta=\alpha k^{-4 l}$. For a set $Z \subset V$ let $X_{Z}$ be the number of indices $i \in[m]$ such that $\boldsymbol{\Phi}_{i}$ is a clause with precisely l positive literals that contains a variable from $Z$. Then $\max \left\{X_{Z}:|Z| \leq \delta n\right\} \leq \sqrt{\delta}$ n w.h.p.

Proof. Let $\mu=\lceil\sqrt{\delta} n\rceil$. We use a first moment argument. Clearly we just need to consider sets $Z$ of size $\lfloor\delta n\rfloor$. Thus, there are at most $\left(\begin{array}{c}n \\ \delta n\end{array}\right)$ ways to choose $Z$. Once $Z$ is fixed, there are at most $\left(\begin{array}{c}m \\ \mu\end{array}\right)$ ways to 
choose a set $\mathcal{I} \subset[m]$ of size $\mu$. For each $i \in \mathcal{I}$ the probability that $\boldsymbol{\Phi}_{i}$ contains a variable from $Z$ and has precisely $l$ positive literals is at most $2^{1-k} k\left(\begin{array}{c}k \\ l\end{array}\right) \delta$ Hence, by the union bound

$$
\begin{aligned}
\mathrm{P}\left[\max \left\{X_{Z}:|Z| \leq \delta n\right\} \geq \mu\right] & \leq\left(\begin{array}{c}
n \\
\delta n
\end{array}\right)\left(\begin{array}{c}
m \\
\mu
\end{array}\right)\left[2^{1-k} k\left(\begin{array}{c}
k \\
l
\end{array}\right) \delta\right]^{\mu} \leq\left(\frac{\mathrm{e}}{\delta}\right)^{\delta n}\left(\frac{2 \mathrm{e} k m\left(\begin{array}{l}
k \\
l
\end{array}\right) \delta}{2^{k} \mu}\right)^{\mu} \\
& \leq\left(\frac{\mathrm{e}}{\delta}\right)^{\delta n}\left(\frac{2 \mathrm{e} \ln (k)\left(\begin{array}{c}
k \\
l
\end{array}\right) \delta n}{\mu}\right)^{\mu} \quad\left[\text { as } m \leq 2^{k} k^{-1} \ln k\right] \\
& \left.\leq\left(\frac{\mathrm{e}}{\delta}\right)^{\delta n}\left(4 \mathrm{e} \ln (k) \cdot k^{l} \cdot \sqrt{\delta}\right)^{\mu} \quad \text { [because } \mu=\lceil\sqrt{\delta} n\rceil\right] \\
& \leq\left(\frac{\mathrm{e}}{\delta}\right)^{\delta n} \delta^{\mu / 8} \quad\left[\text { as } \delta=\alpha k^{-4 l} \text { for a small } \alpha>0\right] \\
& =\exp \left[n \sqrt{\delta}\left(\sqrt{\delta}(1-\ln \delta)+\frac{1}{8} \ln \delta\right)\right] .
\end{aligned}
$$

The last expression is $o(1)$, because $\sqrt{\delta}(1-\ln \delta)+\frac{1}{8} \ln \delta$ is negative for sufficiently small $\delta$.

\section{The algorithm Fix}

In this section we present the algorithm Fix. To establish Theorem 1.1 we will prove the following: for any $0<\varepsilon<0.1$ there is $k_{0}=k_{0}(\varepsilon)>3$ such that for all $k \geq k_{0}$ the algorithm $\mathrm{Fix}$ outputs a satisfying assignment w.h.p. when applied to $\boldsymbol{\Phi}$ with $m=\left\lfloor(1-\varepsilon) 2^{k} k^{-1} \ln k\right\rfloor$. Thus, we assume that $k$ exceeds some large enough number $k_{0}$ depending on $\varepsilon$ only. In addition, we assume throughout that $n>n_{0}$ for some large enough $n_{0}=n_{0}(\varepsilon, k)$. We set

$$
\omega=(1-\varepsilon) \ln k \text { and } k_{1}=\lceil k / 2\rceil .
$$

Let $\Phi \in \Omega_{k}(n, m)$ be a $k$-SAT instance. When applied to $\Phi$ the algorithm basically tries to "fix" the all-true assignment by setting "a few" variables $Z \subset V$ to false so as to satisfy all clauses. Obviously, the set $Z$ will have to contain one variable from each clause consisting of negative literals only. The key issue is to pick "the right" variables. To this end, the algorithm goes over the all-negative clauses in the natural order. If the present all-negative clause $\Phi_{i}$ does not contain a variable from $Z$ yet, $\mathrm{Fix}$ (tries to) identify a "safe" variable in $\Phi_{i}$, which it then adds to $Z$. Here "safe" means that setting the variable to false does not create new unsatisfied clauses. More precisely, we say that a clause $\Phi_{i}$ is $Z$-unique if $\Phi_{i}$ contains exactly one positive literal from $V \backslash Z$ and no negative literal whose underlying variable is in $Z$. Moreover, $x \in V \backslash Z$ is $Z$-unsafe if it occurs positively in a $Z$-unique clause, and $Z$-safe if this is not the case. Then in order to fix an all-negative clause $\Phi_{i}$ we prefer $Z$-safe variables.

To implement this idea, Fix proceeds in three phases. Phase 1 performs the operation described in the previous paragraph: try to identify a $Z$-safe variable in each all-negative clause. Of course, it will happen that an all-negative clause does not contain a $Z$-safe variable. In this case $\mathrm{F}$ ix just picks the variable in position $k_{1}$. Consequently, the assignment constructed in the first phase will not satisfy all clauses. However, we will prove that the number of unsatisfied clauses is very small, and the purpose of Phases 2 and 3 is to deal with them. Before we come to this, let us describe Phase 1 precisely.

Algorithm 3.1 $\operatorname{Fix}(\Phi)$

Input: A $k$-SAT formula $\Phi$. Output: Either a satisfying assignment or "fail".

1a. Let $Z=\emptyset$.

1b. For $i=1, \ldots, m$ do

1c. If $\Phi_{i}$ is all-negative and contains no variable from $Z$

1d. If there is $1 \leq j<k_{1}$ such that $\left|\Phi_{i j}\right|$ is $Z$-safe, then pick the least such $j$ and add $\left|\Phi_{i j}\right|$ to $Z$.

1e. $\quad$ Otherwise add $\left|\Phi_{i k_{1}}\right|$ to $Z$. 
The following proposition, which we will prove in Section 4 summarizes the analysis of Phase 1 . Let $\sigma_{Z}$ be the assignment that sets all variables in $V \backslash Z$ to true and all variables in $Z$ to false.

Proposition 3.2 At the end of the first phase of $F$ ix $(\boldsymbol{\Phi})$ the following statements are true w.h.p.

1. We have $|Z| \leq 4 n k^{-1} \ln \omega$.

2. At most $(1+\varepsilon / 3) \omega n$ clauses are $Z$-unique.

3. At most $\exp \left(-k^{\varepsilon / 8}\right)$ n clauses are unsatisfied under $\sigma_{Z}$.

Since the probability that a random clause is all-negative is $2^{-k}$, under the all-true assignment $(1+$ $o(1)) 2^{-k} m \sim \omega n / k$ clauses are unsatisfied w.h.p. Hence, the outcome $\sigma_{Z}$ of Phase 1 is already a lot better than the all-true assignment w.h.p.

Step $1 \mathrm{~d}$ only considers indices $1 \leq j \leq k_{1}$. This is just for technical reasons, namely to maintain a certain degree of stochastic independence to facilitate (the analysis of) Phase 2.

Phase 2 deals with the clauses that are unsatisfied under $\sigma_{Z}$. The general plan is similar to Phase 1: we (try to) identify a set $Z^{\prime}$ of "safe" variables that can be used to satisfy the $\sigma_{Z}$-unsatisfied clauses without "endangering" further clauses. More precisely, we say that a clause $\Phi_{i}$ is $\left(Z, Z^{\prime}\right)$-endangered if there is no $1 \leq j \leq k$ such that the literal $\Phi_{i j}$ is true under $\sigma_{Z}$ and $\left|\Phi_{i j}\right| \in V \backslash Z^{\prime}$. In words, $\Phi_{i}$ is $\left(Z, Z^{\prime}\right)$-endangered if it relies on one of the variables in $Z^{\prime}$ to be satisfied. Call $\Phi_{i}\left(Z, Z^{\prime}\right)$-secure if it is not $\left(Z, Z^{\prime}\right)$-endangered. Phase 2 will construct a set $Z^{\prime}$ such that for all $1 \leq i \leq m$ one of the following is true:

- $\Phi_{i}$ is $\left(Z, Z^{\prime}\right)$-secure.

- There are at least three indices $1 \leq j \leq k$ such that $\left|\Phi_{i j}\right| \in Z^{\prime}$.

To achieve this, we say that a variable $x$ is $\left(Z, Z^{\prime}\right)$-unsafe if $x \in Z \cup Z^{\prime}$ or there are indices $(i, l) \in[m] \times[k]$ such that the following two conditions hold:

a. For all $j \neq l$ we have $\Phi_{i j} \in Z \cup Z^{\prime} \cup \overline{V \backslash Z}$.

b. $\Phi_{i l}=x$.

(In words, $x$ occurs positively in $\Phi_{i}$, and all other literals of $\Phi_{i}$ are either positive but in $Z \cup Z^{\prime}$ or negative but not in $Z$.) Otherwise we call $x\left(Z, Z^{\prime}\right)$-safe. In the course of the process, Fix greedily tries to add as few $\left(Z, Z^{\prime}\right)$-unsafe variables to $Z^{\prime}$ as possible.

2a. Let $Q$ consist of all $i \in[m]$ such that $\Phi_{i}$ is unsatisfied under $\sigma_{Z}$. Let $Z^{\prime}=\emptyset$.

2b. While $Q \neq \emptyset$

2c. $\quad$ Let $i=\min Q$.

2d. If there are indices $k_{1}<j_{1}<j_{2}<j_{3} \leq k-5$ such that $\left|\Phi_{i j_{l}}\right|$ is $\left(Z, Z^{\prime}\right)$-safe for $l=1,2,3$,

2e. else pick the lexicographically first such sequence and add $\left|\Phi_{i j_{1}}\right|,\left|\Phi_{i j_{2}}\right|,\left|\Phi_{i j_{3}}\right|$ to $Z^{\prime}$.

let $k-5<j_{1}<j_{2}<j_{3} \leq k$ be the lexicographically first sequence such that $\left|\Phi_{i j_{l}}\right| \notin Z$ ' and add $\left|\Phi_{i j_{l}}\right|$ to $Z^{\prime}(l=1,2,3)$.

2f. Let $Q$ be the set of all $\left(Z, Z^{\prime}\right)$-endangered clauses that contain less than 3 variables from $Z^{\prime}$.

Note that the While-loop gets executed at most $n / 3$ times, because $Z^{\prime}$ gains three new elements in each iteration. Actually we prove in Section 5 below that the final set $Z^{\prime}$ is fairly small w.h.p.

Proposition 3.3 The set $Z^{\prime}$ obtained in Phase 2 of $\operatorname{Fix}(\boldsymbol{\Phi})$ has size $\left|Z^{\prime}\right| \leq n k^{-12}$ w.h.p.

After completing Phase 2, Fix is going to set the variables in $V \backslash\left(Z \cup Z^{\prime}\right)$ to true and the variables in $Z \backslash Z^{\prime}$ to false. This will satisfy all $\left(Z, Z^{\prime}\right)$-secure clauses. In order to satisfy the $\left(Z, Z^{\prime}\right)$-endangered clauses as well, Fix needs to set the variables in $Z^{\prime}$ appropriately. Since each $\left(Z, Z^{\prime}\right)$-endangered clauses contains three variables from $Z^{\prime}$, this is essentially equivalent to solving a 3-SAT problem, in which $Z^{\prime}$ is the set of variables. As we shall see, w.h.p. the resulting 3-SAT instance is sufficiently sparse for the following "matching heuristic" to succeed: set up a bipartite graph $G\left(\Phi, Z, Z^{\prime}\right)$ whose vertex set consists of 
the $\left(Z, Z^{\prime}\right)$-endangered clauses and the set $Z^{\prime}$. Each $\left(Z, Z^{\prime}\right)$-endangered clause is adjacent to the variables from $Z^{\prime}$ that occur in it. If there is a matching $M$ in $G\left(\Phi, Z, Z^{\prime}\right)$ that covers all $\left(Z, Z^{\prime}\right)$-endangered clauses, we construct an assignment $\sigma_{Z, Z^{\prime}, M}$ as follows: for each variable $x \in V$ we define

$$
\sigma_{Z, Z^{\prime}, M}(x)= \begin{cases}\text { false } & \text { if } x \in Z \backslash Z^{\prime} \\ \text { false } & \text { if }\left\{\Phi_{i}, x\right\} \in M \text { for some } 1 \leq i \leq m \text { and } x \text { occurs negatively in } \Phi_{i}, \\ \text { true } & \text { otherwise. }\end{cases}
$$

To be precise, Phase 3 proceeds as follows.

3. If $G\left(\Phi, Z, Z^{\prime}\right)$ has a matching that covers all $\left(Z, Z^{\prime}\right)$-endangered clauses, then compute an (arbitrary) such matching $M$ and output $\sigma_{Z, Z^{\prime}, M}$. If not, output "fail".

The (bipartite) matching computation can be performed in $O\left((n+m)^{3 / 2}\right)$ time via the Hopcroft-Karp algorithm. In Section 6 we will show that the matching exists w.h.p.

Proposition 3.4 W.h.p. $G\left(\Phi, Z, Z^{\prime}\right)$ has a matching that covers all $\left(Z, Z^{\prime}\right)$-endangered clauses.

Proof of Theorem 1.1 Fix is clearly a deterministic polynomial time algorithm. It remains to show that $\operatorname{Fix}(\boldsymbol{\Phi})$ outputs a satisfying assignment w.h.p. By Proposition 3.4 Phase 3 will find a matching $M$ that covers all $\left(Z, Z^{\prime}\right)$-endangered clauses w.h.p., and thus the output will be the assignment $\sigma=\sigma_{Z, Z^{\prime}, M}$ w.h.p. Assume that this is the case. Then $\sigma$ sets all variables in $Z \backslash Z^{\prime}$ to false and all variables in $V \backslash\left(Z \cup Z^{\prime}\right)$ to true, thereby satisfying all $\left(Z, Z^{\prime}\right)$-secure clauses. Furthermore, for each $\left(Z, Z^{\prime}\right)$-endangered clause $\boldsymbol{\Phi}_{i}$ there is an edge $\left\{\boldsymbol{\Phi}_{i},\left|\boldsymbol{\Phi}_{i j}\right|\right\}$ in $M$. If $\boldsymbol{\Phi}_{i j}$ is negative, then $\sigma\left(\left|\boldsymbol{\Phi}_{i j}\right|\right)=$ false, and if if $\boldsymbol{\Phi}_{i j}$ is positive, then $\sigma\left(\boldsymbol{\Phi}_{i j}\right)=$ true. In either case $\sigma$ satisfies $\boldsymbol{\Phi}_{i}$.

\section{Proof of Proposition 3.2}

Throughout this section we let $0<\varepsilon<0.1$ and assume that $k \geq k_{0}$ for a sufficiently large $k_{0}=k_{0}(\varepsilon)$. Moreover, we assume that $m=\left\lfloor(1-\varepsilon) 2^{k} k^{-1} \ln k\right\rfloor$ and that $n>n_{0}$ for some large enough $n_{0}=n_{0}(\varepsilon, k)$. Let $\omega=(1-\varepsilon) \ln k$ and $k_{1}=\lceil k / 2\rceil$.

\subsection{Outline}

Before we proceed to the analysis, it is worthwhile giving a brief intuitive explanation as to why Phase 1 "works". Namely, let us just consider the first all-negative clause $\boldsymbol{\Phi}_{i}$ of the random input formula. Without loss of generality we may assume that $i=1$. Given that $\Phi_{1}$ is all-negative, the $k$-tuple of variables $\left(\left|\Phi_{1 j}\right|\right)_{1 \leq j \leq k} \in V^{k}$ is uniformly distributed. Furthermore, at this point $Z=\emptyset$. Hence, a variable $x$ is $Z$-unsafe iff it occurs as the unique positive literal in some clause. The expected number of clauses with exactly one positive literal is $k 2^{-k} m \sim \omega n$. Thus, for each variable $x$ the expected number of clauses in which $x$ is the only positive literal is $k 2^{-k} m / n \sim \omega$. In fact, for each variable the number of such clauses is asymptotically Poisson. Consequently, the probability that $x$ is not $Z$-supporting is $(1+o(1)) \exp (-\omega)$. Returning to the clause $\boldsymbol{\Phi}_{1}$, we conclude that the expected number of indices $1 \leq j \leq k_{1}$ such that $\left|\boldsymbol{\Phi}_{1 j}\right|$ is $Z$-safe is $(1+o(1)) k_{1} \exp (-\omega)$. Since $\omega=(1-\varepsilon) \ln k$, we have

$$
(1+o(1)) k_{1} \exp (-\omega) \geq k^{\varepsilon} / 3 .
$$

Indeed, the number of indices $1 \leq j \leq k_{1}$ so that $\left|\boldsymbol{\Phi}_{1 j}\right|$ is $Z$-safe is binomially distributed, and hence the probability that there is no $Z$-safe $\left|\boldsymbol{\Phi}_{1 j}\right|$ is at most $(1+o(1)) \exp \left(-k^{\varepsilon} / 3\right)$. Thus, it is "quite likely" that $\boldsymbol{\Phi}_{1}$ can be satisfied by setting some variable to false without creating any new unsatisfied clauses. Of course, this argument only applies to the first all-negative clause (i.e., $Z=\emptyset$ ), and the challenge lies in dealing with the stochastic dependencies that arise in the course of the execution.

To this end, we need to investigate how the set $Z$ computed in Steps 1 evolves over time. Thus, we will analyze the execution of Phase 1 as a stochastic process, in which the set $Z$ corresponds to a sequence 
$\left(Z_{t}\right)_{t \geq 0}$ of sets. The time parameter $t$ is the number of all-negative clauses for which either Step $1 \mathrm{~d}$ or $1 \mathrm{e}$ has been executed. We will represent the execution of Phase 1 on input $\Phi$ by a sequence of (random) maps

$$
\pi_{t}:[m] \times[k] \rightarrow\{-1,1\} \cup V \cup \bar{V} .
$$

The map $\pi_{t}$ is meant to capture the information that has determined the first $t$ steps of the process. If $\pi_{t}(i, j)=1$ (resp. $\pi_{t}(i, j)=-1$ ), then $\mathrm{Fix}$ has only taken into account that $\boldsymbol{\Phi}_{i j}$ is a positive (negative) literal, but not what the underlying variable is. If $\pi_{t}(i, j) \in V \cup \bar{V}$, then $\mathrm{Fix}$ has revealed the actual literal $\boldsymbol{\Phi}_{i j}$.

Let us define the sequence $\pi_{t}(i, j)$ precisely. Let $Z_{0}=\emptyset$. Moreover, let $U_{0}$ be the set of all $i$ such that there is exactly one $j$ such that $\boldsymbol{\Phi}_{i j}$ is positive. Further, define $\pi_{0}(i, j)$ for $(i, j) \in[m] \times[k]$ as follows. If $i \in U_{0}$ and $\boldsymbol{\Phi}_{i j}$ is positive, then let $\pi_{0}(i, j)=\boldsymbol{\Phi}_{i j}$. Otherwise, let $\pi_{0}(i, j)$ be 1 if $\boldsymbol{\Phi}_{i j}$ is a positive literal and -1 if $\boldsymbol{\Phi}_{i j}$ is a negative literal. In addition, for $x \in V$ let

$$
U_{0}(x)=\left|\left\{i \in U_{0}: \exists j \in[k]: \pi_{0}(i, j)=x\right\}\right|
$$

be the number of clauses in which $x$ is the unique positive literal. For $t \geq 1$ we define $\pi_{t}$ as follows.

PI1 If there is no index $i \in[m]$ such that $\boldsymbol{\Phi}_{i}$ is all-negative but contains no variable from $Z_{t-1}$, the process stops. Otherwise let $\phi_{t}$ be the smallest such index.

PI2 If there is $1 \leq j<k_{1}$ such that $U_{t-1}\left(\left|\Phi_{\phi_{t}}\right|\right)=0$, then choose the smallest such index; otherwise let $j=k_{1}$. Let $z_{t}=\boldsymbol{\Phi}_{\phi_{t} j_{t}}$ and $Z_{t}=Z_{t-1} \cup\left\{z_{t}\right\}$.

PI3 Let $U_{t}$ be the set of all $i \in[m]$ such that $\boldsymbol{\Phi}_{i}$ is $Z_{t}$-unique. For $x \in V$ let $U_{t}(x)$ be the number of indices $i \in U_{t}$ such that $x$ occurs positively in $\boldsymbol{\Phi}_{i}$.

PI4 For any $(i, j) \in[m] \times[k]$ let

$$
\pi_{t}(i, j)=\left\{\begin{array}{cl}
\boldsymbol{\Phi}_{i j} & \text { if }\left(i=\phi_{t} \wedge j \leq k_{1}\right) \vee\left|\boldsymbol{\Phi}_{i j}\right| \in Z_{t} \vee\left(i \in U_{t} \wedge \pi_{0}(i, j)=1\right), \\
\pi_{t-1}(i, j) & \text { otherwise }
\end{array}\right.
$$

Let $T$ be the total number of iterations of this process before it stops and define $\pi_{t}=\pi_{T}, Z_{t}=Z_{T}$, $U_{t}=U_{T}, U_{t}(x)=U_{T}(x), \phi_{t}=z_{t}=0$ for all $t>T$.

Let us discuss briefly how the above process mirrors Phase 1 of Fix. Step PI1 selects the least index $\phi_{t}$ such that clause $\boldsymbol{\Phi}_{\phi_{t}}$ is all-negative but contains no variable from the $Z_{t-1}$ of variables that have been selected to be set to false so far. In terms of the description of $\mathrm{Fix}$, this corresponds to jumping forward to the next execution of Steps 1d-e. Since $U_{t-1}(x)$ is the number of $Z_{t-1}$-unique clauses in which variable $x$ occurs positively, Step PI2 applies the same rule as $1 \mathrm{~d}-\mathrm{e}$ of $\mathrm{F}$ ix to select the new element $z_{t}$ to be included in the set $Z_{t}$. Step PI3 then "updates" the numbers $U_{t}(x)$. Finally, step PI4 sets up the map $\pi_{t}$ to represent the information that has guided the process so far: we reveal the first $k_{1}$ literals of the current clause $\boldsymbol{\Phi}_{\phi_{t}}$, all occurrences of the variable $z_{t}$, and all positive literals of $Z_{t}$-unique clauses.

Observe that at each time $t \leq T$ the process PI1-PI4 adds precisely one variable $z_{t}$ to $Z_{t}$. Thus, $\left|Z_{t}\right|=t$ for any $t \leq T$. Furthermore, for $1 \leq t \leq T$ the map $\pi_{t}$ is obtained from $\pi_{t-1}$ by replacing some \pm 1 s by literals, but no changes of the opposite type are made. Finally, for any $i \in[m]$ there is either no $j$ such that $\pi_{t}(i, j)=1$, or there are at least two such indices $j$. This is because step PI4 ensures that for any $i$ such that $\boldsymbol{\Phi}_{i}$ is $Z_{t}$-unique $\pi_{t}(i, j)$ equals the literal $\boldsymbol{\Phi}_{i j}$ if it is positive.

Of course, the process PI1-PI4 can be applied to any concrete $k$-SAT formula $\Phi$ (rather than the random $\boldsymbol{\Phi})$. It then yields a sequence $\pi_{t}[\Phi]$ of maps, variables $z_{t}[\Phi]$, etc.

For each integer $t \geq 0$ we define an equivalence relation $\equiv_{t}$ on the set $\Omega_{k}(n, m)$ of $k$-SAT formulas by letting $\Phi \equiv_{t} \Psi$ iff $\pi_{s}[\Phi]=\pi_{s}[\Psi]$ for all $0 \leq s \leq t$. Let $\mathcal{F}_{t}$ be the $\sigma$-algebra generated by the equivalence classes of $\equiv_{t}$. The family $\left(\mathcal{F}_{t}\right)_{t \geq 0}$ is a filtration, and the following is immediate from the construction.

Fact 4.1 For any $t \geq 0$ the random map $\pi_{t}$, the random variables $\phi_{t+1}$ and $z_{t}$, the random sets $U_{t}$ and $Z_{t}$, and the random variables $U_{t}(x)$ for $x \in V$ are $\mathcal{F}_{t}$-measurable.

Intuitively, that a random variable $X$ is $\mathcal{F}_{t}$-measurable means that its value is determined by time $t$. The following is the key fact about the sequence $\pi_{t}(i, j)$. 
Proposition 4.2 Let $\mathcal{E}_{t}$ be the set of all pairs $(i, j)$ such that $\pi_{t}(i, j) \in\{-1,1\}$. The conditional joint distribution of the variables $\left(\left|\Phi_{i j}\right|\right)_{(i, j) \in \mathcal{E}_{t}}$ given $\mathcal{F}_{t}$ is uniform over $\left(V \backslash Z_{t}\right)^{\mathcal{E}_{t}}$. That is, for any formula $\Phi$ and for any map $f$ from $\mathcal{E}_{t}[\Phi]$ to $V \backslash Z_{t}[\Phi]$ we have

$$
\mathrm{P}\left[\forall(i, j) \in \mathcal{E}_{t}[\Phi]:\left|\boldsymbol{\Phi}_{i j}\right|=f(i, j) \mid \mathcal{F}_{t}\right](\Phi)=\left|V \backslash Z_{t}[\Phi]\right|^{-\left|\mathcal{E}_{t}[\Phi]\right|} .
$$

Proof. Let $[\Phi]_{t}$ signify the $\equiv_{t}$-equivalence class of $\Phi$. Let $\mathrm{P}_{\Phi}$ denote the conditional probability distribution $\mathrm{P}\left[\cdot \mid \mathcal{F}_{t}\right](\Phi)$. Then for any event $X$ we have

$$
\mathrm{P}_{\Phi}[X]=\mathrm{P}\left[X \mid[\Phi]_{t}\right]=\left|[\Phi]_{t} \cap X\right| /\left|[\Phi]_{t}\right| .
$$

That is, the conditional distribution $\mathrm{P}_{\Phi}$ is uniform over $[\Phi]_{t}$. Hence, we just need to determine $\left|[\Phi]_{t}\right|$. Given a map $f: \mathcal{E}_{t}[\Phi] \rightarrow V \backslash Z_{t}[\Phi]$, we define a formula $\Phi_{f}$ by letting

$$
\left(\Phi_{f}\right)_{i j}=\left\{\begin{array}{cl}
\overline{f(i, j)} & \text { if }(i, j) \in \mathcal{E}_{t}[\Phi] \text { and } \pi_{0}(i, j)=-1, \\
f(i, j) & \text { if }(i, j) \in \mathcal{E}_{t}[\Phi] \text { and } \pi_{0}(i, j)=1, \\
\Phi_{i j} & \text { otherwise }
\end{array} \quad(i \in[m], j \in[k]) .\right.
$$

Then $\Phi_{f} \equiv_{t} \Phi$. Hence, we obtain a bijection $\left(V \backslash Z_{t}[\Phi]\right)^{\mathcal{E}_{t}[\Phi]} \rightarrow[\Phi]_{t}, f \mapsto \Phi_{f}$, and thus the assertion follows from (3).

In each step of the process PI1-PI4 one variable $z_{t}$ is added to $Z_{t}$. There is a chance that this variable occurs in several other all-negative clauses, and therefore the stopping time $T$ should be smaller than the total number of all-negative clauses. To prove this, we need the following lemma.

Lemma 4.3 W.h.p. the following is true for all $1 \leq t \leq \min \{T, n\}$ : the number of indices $i \in[m]$ such that $\pi_{t}(i, j)=-1$ for all $1 \leq j \leq k$ is at most $2 n \omega \exp (-k t / n) / k$.

Proof. We consider the random variables

$$
\mathcal{N}_{t i j}=\left\{\begin{array}{ll}
1 & \text { if } \pi_{t}(i, j)=-1 \text { and } t \leq T, \\
0 & \text { otherwise }
\end{array} \quad(i \in[m], j \in[k], t \geq 0) .\right.
$$

Let $t \leq n, \mu=\left\lceil\ln ^{2} n\right\rceil$, and let $\mathcal{I} \subset[m]$ be a set of size $\mu$. Let $Y_{i}=1$ if $t \leq T$ and $\pi_{t}(i, j)=-1$ for all $j \in[k]$, and let $Y_{i}=0$ otherwise. Set $\mathcal{J}=[t] \times \mathcal{I} \times[k]$. If $Y_{i}=1$ for all $i \in \mathcal{I}$, then $\mathcal{N}_{0 i j}=1$ for all $(i, j) \in \mathcal{I} \times[k]$ and $\mathcal{N}_{s i j}=1$ for all $(s, i, j) \in \mathcal{J}$, and we will prove below that

$$
\mathrm{E}\left[\prod_{(i, j) \in \mathcal{I} \times[k]} \mathcal{N}_{0 i j} \cdot \prod_{(t, i, j) \in \mathcal{J}} \mathcal{N}_{t i j}\right] \leq 2^{-k|\mathcal{I}|}(1-1 / n)^{|\mathcal{J}|} .
$$

Hence,

$$
\mathrm{E}\left[\prod_{i \in \mathcal{I}} Y_{i}\right] \leq\left[2^{-k}(1-1 / n)^{k t}\right]^{\mu} \leq \lambda^{\mu}, \quad \text { where } \lambda=2^{-k} \exp (-k t / n)
$$

Combining the bound (5) with Lemma 2.2 we see that with probability at least $1-n^{-10}$ there are no more than $2 \lambda m$ indices $i \in[m]$ such that $\pi_{t}(i, j)=-1$ for all $j \in[k]$. Hence, by the union bound the probability that this holds for all $t \leq \min \{T, n\}$ is at least $1-n^{-9}$. As $2 \lambda m \leq 2 n \omega \exp (-k t / n) / k$, this implies the assertion.

To complete the proof, we need to establish (4). Let

$$
X_{0}=\prod_{(i, j) \in \mathcal{I} \times[k]} \mathcal{N}_{0 i j}, \mathcal{J}_{t}=\{(i, j):(t, i, j) \in \mathcal{J}\}, \text { and } X_{t}=\prod_{(i, j) \in \mathcal{J}_{t}} \mathcal{N}_{t i j} .
$$

Since the signs of the literals $\boldsymbol{\Phi}_{i j}$ are mutually independent, we have

$$
\mathrm{E}\left[X_{0}\right]=2^{-k|\mathcal{I}|}
$$


Furthermore, we claim that

$$
\mathrm{E}\left[X_{t} \mid \mathcal{F}_{t-1}\right] \leq(1-1 / n)^{\left|\mathcal{J}_{t}\right|}
$$

then (4) follows by plugging (6) and (7) into Lemma 2.4

To prove (7), let $t \geq 1$. If $T<t$ or $\pi_{t-1}(i, j) \neq-1$ for some $(i, j) \in \mathcal{J}_{t}$, then clearly $X_{t}=\mathcal{N}_{t i j}=0$. Hence, suppose that $T \geq t$ and $\pi_{t-1}(i, j)=-1$ for all $(i, j) \in \mathcal{J}_{t}$. Then at time $t$ PI2 selects some variable $z_{t} \in V \backslash Z_{t-1}$, and $\mathcal{N}_{t i j}=1$ only if $\left|\boldsymbol{\Phi}_{i j}\right| \neq z_{t}$. As $\pi_{t-1}(i, j)=-1$ for all $(i, j) \in \mathcal{J}_{t}$, given $\mathcal{F}_{t-1}$ the variables $\left(\left|\boldsymbol{\Phi}_{i j}\right|\right)_{(i, j) \in \mathcal{J}_{t}}$ are mutually independent and uniformly distributed over $V \backslash Z_{t-1}$ by Proposition 4.2 Therefore, for each $(i, j) \in \mathcal{J}_{t}$ independently we have $\left|\boldsymbol{\Phi}_{i j}\right|=z_{t}$ with probability at least $1 / n$, whence (7) follows.

Corollary 4.4 W.h.p. we have $T<4 n k^{-1} \ln \omega$.

Proof. Let $t_{0}=2 n k^{-1} \ln \omega$ and let $I_{t}$ be the number of indices $i$ such that $\pi_{t}(i, j)=-1$ for all $1 \leq j \leq k$. Then PI2 ensures that $I_{t} \leq I_{t-1}-1$ for all $t \leq T$. Consequently, if $T \geq 2 t_{0}$, then $0 \leq I_{T} \leq I_{t_{0}}-t_{0}$, and thus $I_{t_{0}} \geq t_{0}$. Since $2 n k^{-1} \ln \omega>3 n \omega \exp \left(-k t_{0} / n\right) / k$ for sufficiently large $k$, Lemma 4.3 entails that

$$
\mathrm{P}\left[T \geq 2 t_{0}\right] \leq \mathrm{P}\left[I_{t_{0}} \geq t_{0}\right]=\mathrm{P}\left[I_{t_{0}} \geq 2 n k^{-1} \ln \omega\right] \leq \mathrm{P}\left[I_{t_{0}}>3 n \omega \exp \left(-k t_{0} / n\right) / k\right]=o(1) .
$$

Hence, $T<2 t_{0}$ w.h.p.

$\square$ For the rest of this section we let

$$
\theta=\left\lfloor 4 n k^{-1} \ln \omega\right\rfloor
$$

The next goal is to estimate the number of $Z_{t}$-unique clauses, i.e., the size of the set $U_{t}$. For technical reasons we will consider a slightly bigger set: let $\mathcal{U}_{t}$ be the set of all $i \in[m]$ such that there is an index $j$ such that $\pi_{0}(i, j) \neq-1$ but there exists no $j$ such that $\pi_{t}(i, j) \in\{1\} \cup \bar{Z}_{t}$. That is, clause $\boldsymbol{\Phi}_{i}$ contains a positive literal, but by time $t$ there is at most one positive literal $\boldsymbol{\Phi}_{i j}$ left that does not belong to $Z_{t}$, and $\boldsymbol{\Phi}_{i}$ has no negative literal whose underlying variable lies in $Z_{t}$. In Section 4.2 we will establish the following bound.

Lemma 4.5 W.h.p. we have $\max _{0 \leq t \leq T}\left|\mathcal{U}_{t}\right| \leq(1+\varepsilon / 3) \omega n$.

Let us think of the variables $x \in V \backslash Z_{t}$ as "bins" and of the clauses $\boldsymbol{\Phi}_{i}$ with $i \in U_{t}$ as "balls". If we place each ball $i$ into the (unique) bin $x$ such that $x$ occurs positively in $\boldsymbol{\Phi}_{i}$, then by Lemma 4.5 the average number of balls in a bin is at most

$$
\frac{(1+\varepsilon / 3) \omega n}{\left|V \backslash Z_{t}\right|}=\frac{(1+\varepsilon / 3) \omega}{1-t / n} \quad \text { w.h.p. }
$$

As $\omega \leq(1-\varepsilon) \ln k$ and $t \leq T \leq 4 n k^{-1} \ln \omega$ w.h.p. by Corollary 4.4, for large enough $k$ we have $(1+\varepsilon / 3)(1-t / n)^{-1} \omega \leq(1-0.6 \varepsilon) \ln k$. Hence, if the "balls" were uniformly distributed over the "bins", we would expect

$$
\left|V \backslash Z_{t}\right| \exp \left(-\left|U_{t}\right| /\left|V \backslash Z_{t}\right|\right) \geq(n-t) k^{0.6 \varepsilon-1} \geq n k^{\varepsilon / 2-1}
$$

"bins" to be empty. The next corollary shows that this is actually true. We defer the proof to Section 4.3

Corollary 4.6 Let $\mathcal{Q}_{t}=\left|\left\{x \in V \backslash Z_{t}: U_{t}(x)=0\right\}\right|$. Then $\min _{t \leq T} \mathcal{Q}_{t} \geq n k^{\varepsilon / 2-1}$ w.h.p.

Now that we know that there are "a lot" of variables $x \in V \backslash Z_{t-1}$ such that $U_{t}(x)=0$ w.h.p., we can prove that it is quite likely that clause $\boldsymbol{\Phi}_{\phi_{t}}$ contains one. More precisely, we have the following.

\section{Corollary 4.7 Let}

$$
\mathcal{B}_{t}= \begin{cases}1 & \text { if } \min _{1 \leq j<k_{1}} U_{t-1}\left(\left|\boldsymbol{\Phi}_{\phi_{t} j}\right|\right)>0, \mathcal{Q}_{t-1} \geq n k^{\varepsilon / 2-1},\left|U_{t}\right| \leq(1+\varepsilon / 3) \omega n, \text { and } T \geq t \\ 0 & \text { otherwise. }\end{cases}
$$

Then $\mathcal{B}_{t}$ is $\mathcal{F}_{t}$-measurable and $\mathrm{E}\left[\mathcal{B}_{t} \mid \mathcal{F}_{t-1}\right] \leq \exp \left(-k^{\varepsilon / 6}\right)$ for all $1 \leq t \leq \theta$. 
Proof. Since the event $T<t$ and the random variable $\mathcal{Q}_{t-1}$ are $\mathcal{F}_{t-1}$-measurable and as $U_{t-1}\left(\left|\boldsymbol{\Phi}_{\phi_{t} j}\right|\right)$ is $\mathcal{F}_{t}$-measurable for any $j<k_{1}$ by Fact $4.1, \mathcal{B}_{t}$ is $\mathcal{F}_{t}$-measurable. Let $\Phi$ be such that $T[\Phi] \geq t, \mathcal{Q}_{t-1}[\Phi] \geq$ $n k^{\alpha-1}$, and $\left|U_{t-1}[\Phi]\right| \leq(1+\varepsilon / 3) \omega n$. We condition on the event $\Phi \equiv_{t-1} \Phi$. Then at time $t$ the process PI1-PI4 selects $\phi_{t}$ such that $\pi_{t-1}\left(\phi_{t}, j\right)=-1$ for all $j \in[k]$. Hence, by Proposition 4.2 the variables $\left|\boldsymbol{\Phi}_{\phi_{t} j}\right|$ are uniformly distributed and mutually independent elements of $V \backslash Z_{t-1}$. Consequently, for each $j<k_{1}$ the event $U_{t-1}\left(\left|\boldsymbol{\Phi}_{\phi_{t} j}\right|\right)=0$ occurs with probability $\left|\mathcal{Q}_{t-1}\right| /\left|V \backslash Z_{t-1}\right| \geq k^{\varepsilon / 2-1}$ independently. Thus, the probability that $U_{t-1}\left(\left|\boldsymbol{\Phi}_{\phi_{t} j}\right|\right)>0$ for all $j<k_{1}$ is at most $\left(1-k^{\varepsilon / 2-1}\right)^{k_{1}-1} \leq \exp \left(-k^{\varepsilon / 6}\right)$. $\square$

Proof of Proposition 3.2 The definition of the process PI1-PI4 mirrors the execution of the algorithm, i.e., the set $Z$ obtained after Steps 1a-1d of $\mathrm{F}$ ix equals the set $Z_{T}$. Therefore, the first item of Proposition 3.2 is an immediate consequence of Corollary 4.4 and the fact that $\left|Z_{t}\right|=t$ for all $t \leq T$. Furthermore, the second assertion follows directly from Lemma 4.5

To prove the third claim, we need to bound the number of clauses that are unsatisfied under the assignment $\sigma_{Z_{T}}$ that sets all variables in $V \backslash Z_{T}$ to true and all variables in $Z_{T}$ to false. By construction any all-negative clause contains a variable from $Z_{T}$ and is thus satisfied under $\sigma_{Z_{T}}$. We claim that for any $i \in[m]$ such that $\boldsymbol{\Phi}_{i}$ is unsatisfied under $\sigma_{Z_{T}}$ one of the following is true.

a. There is $t \leq T$ such that $i \in U_{t-1}$ and $z_{t}$ occurs positively in $\boldsymbol{\Phi}_{i}$.

b. There are $1 \leq j_{1}<j_{2} \leq k$ such that $\boldsymbol{\Phi}_{i j_{1}}=\boldsymbol{\Phi}_{i j_{2}}$.

To see this, assume that b. does not occur. Let us assume without loss of generality that $\boldsymbol{\Phi}_{i 1}, \ldots, \boldsymbol{\Phi}_{i l}$ are positive and $\boldsymbol{\Phi}_{i l+1}, \ldots, \boldsymbol{\Phi}_{i k}$ are negative for some $l \geq 1$. Since $\boldsymbol{\Phi}_{i}$ is unsatisfied under $\sigma_{Z_{T}}$, we have $\boldsymbol{\Phi}_{i 1}, \ldots, \boldsymbol{\Phi}_{i l} \in Z_{T}$. Hence, for each $1 \leq j \leq l$ there is $t_{j} \leq T$ such that $\boldsymbol{\Phi}_{i j}=z_{t_{j}}$. As $\boldsymbol{\Phi}_{i 1}, \ldots, \boldsymbol{\Phi}_{i k}$ are distinct, the indices $t_{1}, \ldots, t_{l}$ are mutually distinct, too. Assume that $t_{1}<\cdots<t_{l}$, and let $t_{0}=0$. Then $\boldsymbol{\Phi}_{i}$ contains precisely one positive literal from $V \backslash Z_{t_{l-1}}$. Hence, $i \in U_{t_{l-1}}$. Since $\boldsymbol{\Phi}_{i}$ is unsatisfied under $\sigma_{Z_{T}}$ no variable from $Z_{T}$ occurs negatively in $\Phi_{i}$ and thus $i \in U_{s}$ for all $t_{l-1} \leq s<t_{l}$. Therefore, $i \in U_{t_{l}-1}$ and $z_{t_{l}}=\boldsymbol{\Phi}_{i l}$, i.e., a. occurs.

Let $\mathcal{X}$ be the number of indices $i \in[m]$ such that a. occurs. We claim that

$$
\mathcal{X} \leq n \exp \left(-k^{\varepsilon / 7}\right) \quad \text { w.h.p. }
$$

Since the number of $i \in[m]$ for which b. occurs is $O(\ln n)$ w.h.p. by Lemma 2.3, (8) implies the third assertion.

To establish (8), let $\mathcal{B}_{t}$ be as in Corollary 4.7 and set

$$
\mathcal{D}_{t}=\left\{\begin{array}{cl}
U_{t-1}\left(z_{t}\right) & \text { if } \mathcal{B}_{t}=1 \text { and } U_{t-1}\left(z_{t}\right) \leq \ln ^{2} n \\
0 & \text { otherwise }
\end{array}\right.
$$

Then by the definition of the random variables $\mathcal{B}_{t}, \mathcal{D}_{t}$ either

$$
\mathcal{X} \leq \sum_{1 \leq t \leq \theta} \mathcal{D}_{t}
$$

or one of the following events occurs:

i. $T>\theta$.

ii. $\mathcal{Q}_{t}<n k^{\varepsilon / 2-1}$ for some $0 \leq t \leq T$.

iii. $\left|U_{t}\right|>(1+\varepsilon / 3) \omega n$ for some $1 \leq t \leq T$.

iv. $\left|U_{t-1}\left(z_{t}\right)\right|>\ln ^{2} n$ for some $1 \leq t \leq \theta$.

The probability of i. is $o(1)$ by Corollary 4.4 Moreover, ii. does not occur w.h.p. by Corollary 4.6 and the probability of iii. is $o(1)$ by Lemma4.5 If iv. occurs, then the variable $z_{t}$ occurs in at least $\ln ^{2} n$ clauses for some $1 \leq t \leq \theta$, which has probability $o(1)$ by Lemma 2.3 Hence, 91 is true w.h.p.

Thus, we need to bound $\sum_{1 \leq t \leq \theta} \mathcal{D}_{t}$. The random variable $\mathcal{D}_{t}$ is $\mathcal{F}_{t}$-measurable and $\mathcal{D}_{t}=0$ for all $t>\theta$. Let $\overline{\mathcal{D}}_{t}=\mathrm{E}\left[\mathcal{D}_{t} \mid \mathcal{F}_{t-1}\right]$ and $\mathcal{M}_{t}=\sum_{s=1}^{t} \mathcal{D}_{s}-\overline{\mathcal{D}}_{s}$. Then $\left(\mathcal{M}_{t}\right)_{1 \leq t \leq \theta}$ is a martingale. As all 
increments $\mathcal{D}_{s}-\overline{\mathcal{D}}_{s}$ are less than $\ln ^{2} n$ in absolute value by the definition of $\mathcal{D}_{t}$, Lemma 2.5 (AzumaHoeffding) entails that $\mathcal{M}_{\theta}=o(n)$ w.h.p. Hence, w.h.p. we have

$$
\sum_{1 \leq t \leq \theta} \mathcal{D}_{t}=o(n)+\sum_{1 \leq t \leq \theta} \overline{\mathcal{D}}_{t}
$$

We claim that

$$
\overline{\mathcal{D}}_{t} \leq 2 \omega \exp \left(-k^{\varepsilon / 6}\right) \quad \text { for all } 1 \leq t \leq \theta .
$$

For by Corollary 4.7 we have

$$
\mathrm{E}\left[\mathcal{B}_{t} \mid \mathcal{F}_{t-1}\right] \leq \exp \left(-k^{\varepsilon / 6}\right) .
$$

Moreover, given $\mathcal{F}_{t-1}$ we have $\pi_{t-1}\left(\phi_{t}, k_{1}\right)=-1$, whence $z_{t}$ is uniformly distributed over $V \backslash Z_{t-1}$ by Proposition 4.2 Since $\mathcal{B}_{t}=1$ implies $\left|U_{t-1}\right| \leq(1+\varepsilon / 3) \omega n$, this means that the conditional expectation of $U_{t-1}\left(z_{t}\right)$ is at most

$$
\left|U_{t-1}\right| /\left|V \backslash Z_{t-1}\right| \leq \frac{(1+\varepsilon / 3) \omega n}{n-t} \leq 2 \omega .
$$

Combining (12) and (13), we obtain (11). Further, plugging (11) into (10), we get

$$
\sum_{1 \leq t \leq \theta} \mathcal{D}_{t}=2 \omega \exp \left(-k^{\varepsilon / 2} / 3\right) \theta+o(n) \leq 3 \omega \exp \left(-k^{\varepsilon / 6}\right) \theta \leq n \exp \left(-k^{\varepsilon / 7}\right) \quad \text { w.h.p. }
$$

Thus, (8) follows from (9).

\subsection{Proof of Lemma 4.5}

For integers $t \geq 1, i \in[m], j \in[k]$ let

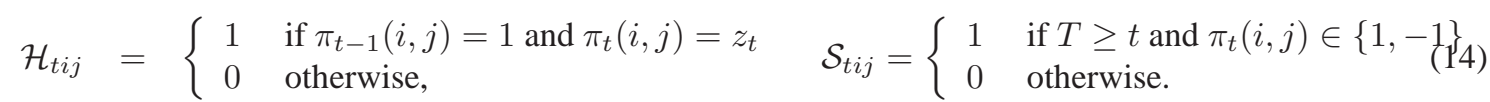

Lemma 4.8 For any two sets $\mathcal{I}, \mathcal{J} \subset[\theta] \times[m] \times[k]$ we have

$$
\mathrm{E}\left[\prod_{(t, i, j) \in \mathcal{I}} \mathcal{H}_{t i j} \cdot \prod_{(t, i, j) \in \mathcal{J}} \mathcal{S}_{t i j} \mid \mathcal{F}_{0}\right] \leq(n-\theta)^{-|\mathcal{I}|}(1-1 / n)^{|\mathcal{J}|}
$$

Proof. Let $\mathcal{I}_{t}=\{(i, j):(t, i, j) \in \mathcal{I}\}, \mathcal{J}_{t}=\{(i, j):(t, i, j) \in \mathcal{J}\}, X_{t}=\prod_{(i, j) \in \mathcal{I}_{t}} \mathcal{H}_{t i j} \prod_{(i, j) \in \mathcal{J}_{t}} \mathcal{S}_{t i j}$. If $X_{t}=1$, then $t \leq T$ (as otherwise $\mathcal{S}_{t i j}=0$ by definition and $\mathcal{H}_{t i j}=0$ because $\pi_{t}=\pi_{t-1}$ ). Furthermore, $X_{t}=1$ implies that

$$
\pi_{t-1}(i, j)=1 \text { for all }(i, j) \in \mathcal{I}_{t} \text { and } \pi_{t-1}(i, j) \in\{-1,1\} \text { for all }(i, j) \in \mathcal{J}_{t} .
$$

Thus, let $\Phi$ be a $k$-SAT formula such that $T[\Phi] \geq t$ and $\pi_{t-1}[\Phi]$ satisfies $[15$. We claim that

$$
\mathrm{E}\left[X_{t} \mid \mathcal{F}_{t-1}\right](\Phi) \leq(n-\theta)^{-\left|\mathcal{I}_{t}\right|}(1-1 / n)^{\left|\mathcal{J}_{t}\right|}
$$

To show this, we condition on the event $\boldsymbol{\Phi} \equiv_{t} \Phi$. Then at time $t$ steps PI1-PI2 select a variable $z_{t}$ from the the all-negative clause $\boldsymbol{\Phi}_{\phi_{t}}$. As for each $(i, j) \in \mathcal{I}_{t}$ clause $\boldsymbol{\Phi}_{i}$ contains a positive literal, we have $\phi_{t} \neq i$. Furthermore, we may assume that if $\left(\phi_{t}, j\right) \in \mathcal{J}_{t}$ then $j>k_{1}$, because otherwise $X_{t}=\mathcal{S}_{t \phi_{t} j}=0$ (cf. PI4). Hence, due to (15) and Proposition 4.2 in the conditional distribution $\mathrm{P}\left[\cdot \mid \mathcal{F}_{t-1}\right](\Phi)$ the variables $\left(\left|\boldsymbol{\Phi}_{i j}\right|\right)_{(i, j) \in \mathcal{I}_{t} \cup \mathcal{J}_{t}}$ are uniformly distributed over $V \backslash Z_{t-1}$ and mutually independent. Therefore, the events $\left|\boldsymbol{\Phi}_{i j}\right|=z_{t}$ occur independently with probability $1 /\left|V \backslash Z_{t-1}\right|=1 /(n-t+1)$, whence

$$
\mathrm{E}\left[X_{t} \mid \mathcal{F}_{t-1}\right](\Phi) \leq(n-t+1)^{-\left|\mathcal{I}_{t}\right|}(1-1 /(n-t+1))^{\left|\mathcal{J}_{t}\right|} \leq(n-\theta)^{-\left|\mathcal{I}_{t}\right|}(1-1 / n)^{\left|\mathcal{J}_{t}\right|} .
$$

This shows (16). Finally, the assertion follows from Lemma 2.4 and (16). $\square$ Armed with Lemma 4.8, we can now bound the number of indices $i \in \mathcal{U}_{t}$ such that $\boldsymbol{\Phi}_{i}$ has "few" positive literals. 
Lemma 4.9 With probability $1-o(1 / n)$ the following is true for all $1 \leq l<\sqrt{k}$ and all $1 \leq t \leq$ $\min \{T, \theta\}$. Let

$$
\Lambda_{l}(t)=\omega\left(\begin{array}{l}
k-1 \\
l-1
\end{array}\right)\left(\frac{t}{n}\right)^{l-1}(1-t / n)^{k-l} .
$$

There are at most $(1+\varepsilon / 9) \Lambda_{l}(t) n$ indices $i \in \mathcal{U}_{t}$ such that $\boldsymbol{\Phi}_{i}$ has precisely l positive literals.

Proof. Let $\mathcal{M} \subset[m]$ be a set of size $\mu=\left\lceil\ln ^{2} n\right\rceil$ and let $P_{i} \subset[k]$ be a set of size $l-1$ for each $i \in \mathcal{M}$. Let $\mathcal{P}=\left(P_{i}\right)_{i \in \mathcal{M}}$ be the family of all sets $P_{i}$. Furthermore, let $t_{i}: P_{i} \rightarrow[t]$ for all $i \in \mathcal{M}$, and let $\mathcal{T}=\left(t_{i}\right)_{i \in \mathcal{M}}$ comprise all maps $t_{i}$. Let $\mathcal{E}_{\mathcal{M}}(\mathcal{P}, \mathcal{T})$ be the event that the following statements are true:

a. $\boldsymbol{\Phi}_{i}$ has exactly $l$ positive literals for all $i \in \mathcal{M}$.

b. $\Phi_{i j}=z_{t_{i}(j)}$ for all $i \in \mathcal{M}$ and $j \in P_{i}$.

c. $t \leq T$ and no variable from $Z_{t}$ occurs negatively in $\boldsymbol{\Phi}_{i}$.

Moreover, let

$$
\begin{aligned}
& \mathcal{I}=\mathcal{I}_{\mathcal{M}}(\mathcal{P}, \mathcal{T})=\left\{(s, i, j): i \in \mathcal{M}, j \in P_{i}, s=t_{i}(j)\right\}, \\
& \mathcal{J}=\mathcal{J}_{\mathcal{M}}(\mathcal{P}, \mathcal{T})=\left\{(s, i, j): i \in \mathcal{M}, j \in[k] \backslash P_{i}\right\}
\end{aligned}
$$

Let $Y_{i}=1$ if clause $\boldsymbol{\Phi}_{i}$ has exactly $l$ positive literals, including the literals $\boldsymbol{\Phi}_{i j}$ for $j \in P_{i}(i \in \mathcal{M})$. Then $\mathrm{P}\left[Y_{i}=1\right]=(k-l+1) 2^{-k}$ for each $i \in \mathcal{M}$. Moreover, the events $Y_{i}=1$ are mutually independent and $\mathcal{F}_{0}$-measurable. Therefore, by Lemma 4.8

$$
\begin{aligned}
\mathrm{P}\left[\mathcal{E}_{\mathcal{M}}(\mathcal{P}, \mathcal{T})\right] & \leq \mathrm{E}\left[\prod_{i \in \mathcal{M}} Y_{i}\right] \cdot \mathrm{E}\left[\prod_{(t, i, j) \in \mathcal{I}} \mathcal{H}_{t i j} \cdot \prod_{(t, i, j) \in \mathcal{J}} \mathcal{S}_{t i j} \mid \mathcal{F}_{0}\right] \\
& \leq\left[\frac{k-l+1}{2^{k}} \cdot(n-t)^{1-l}(1-1 / n)^{(k-l+1) t}\right]^{\mu} .
\end{aligned}
$$

Let $\mathcal{E}_{\mathcal{M}}$ be the event that $t \leq T$ and $\boldsymbol{\Phi}_{i}$ has exactly $l$ positive literals and $i \in \mathcal{U}_{t}$ for all $i \in \mathcal{M}$. If $\mathcal{E}_{\mathcal{M}}$ occurs, then there exist $\mathcal{P}, \mathcal{T}$ such that $\mathcal{E}_{\mathcal{M}}(\mathcal{P}, \mathcal{T})$ occurs. Furthermore, for each $i \in \mathcal{M}$ there are $\left(\begin{array}{l}k \\ l-1\end{array}\right)$ ways to choose a set $P_{i}$ and then $t^{l-1}$ ways to choose the map $t_{i}$. Therefore, the union bound and (17) yield

$$
\begin{aligned}
\mathrm{P}\left[\mathcal{E}_{\mathcal{M}}\right] & \leq \sum_{\mathcal{P}, \mathcal{T}} \mathrm{P}\left[\mathcal{E}_{\mathcal{M}}(\mathcal{P}, \mathcal{T})\right] \leq \lambda^{\mu} \quad \text { where } \\
\lambda & =\left(\begin{array}{c}
k \\
l-1
\end{array}\right) t^{l-1} \times \frac{k-l+1}{2^{k}} \cdot(n-t)^{1-l}(1-1 / n)^{(k-l+1) t} .
\end{aligned}
$$

Hence, by Lemma 2.2 with probability $1-o(1 / n)$ there are at most $(1+o(1)) \lambda m$ indices $i \in[m]$ such that $\boldsymbol{\Phi}_{i}$ has precisely $l$ positive literals and $i \in \mathcal{U}_{t}$. Thus, the remaining task is to show that

$$
\lambda m \leq(1+\varepsilon / 10) \Lambda_{l} n .
$$

To show (18), we estimate

$$
\begin{aligned}
\lambda & \leq k 2^{-k} \cdot\left(\begin{array}{c}
k-1 \\
l-1
\end{array}\right)\left(\frac{t}{n-t}\right)^{l-1}(1-1 / n)^{t(k-1-(l-1))} \\
& \leq k 2^{-k} \cdot\left(\begin{array}{c}
k-1 \\
l-1
\end{array}\right)\left(\frac{t}{n}\right)^{l-1}(1-t / n)^{k-1-(l-1)} \eta, \text { where } \eta=\left(\frac{n}{n-t}\right)^{l-1} \cdot\left(\frac{(1-1 / n)^{t}}{1-t / n}\right)^{k-l}(\text {.19) }
\end{aligned}
$$

We can bound $\eta$ as follows:

$$
\begin{aligned}
\eta & \leq(1+t /(n-t))^{l}\left(\frac{\exp (-t / n)}{\exp \left(-t / n-(t / n)^{2}\right)}\right)^{k-l} \leq(1+2 t / n)^{l} \exp \left(k(t / n)^{2}\right) \\
& \leq \exp \left(2 l \theta / n+k(\theta / n)^{2}\right) \leq \exp \left(8 l k^{-1} \ln \omega+16 k^{-1} \ln ^{2} \omega\right) .
\end{aligned}
$$


Since $l \leq \sqrt{k}$ and $\omega \leq \ln k$, the last expression is less than $1+\varepsilon / 10$ for sufficiently large $k$. Hence, $\eta \leq 1+\varepsilon / 10$, and thus $(18)$ follows from $[19] . \quad \square$ The following lemma deals with $i \in \mathcal{U}_{t}$ such that $\boldsymbol{\Phi}_{i}$ contains "a lot" of positive literals.

Lemma 4.10 W.h.p. the following is true for all $l \geq \ln k$. There are at most $n \exp (-l)$ indices $i \in[m]$ such that $\boldsymbol{\Phi}_{i}$ has exactly l positive literals among which at least $l-1$ are in $Z_{\theta}$.

Proof. Let $\mathcal{M} \subset[m]$ be a set of size $\mu=\left\lceil\ln ^{2} n\right\rceil$ and let $P_{i} \subset[k]$ be a set of size $l-1$ for each $i \in \mathcal{M}$. Furthermore, let $t_{i}: P_{i} \rightarrow[\theta]$ for all $i \in \mathcal{M}$, and set $\mathcal{T}=\left(t_{i}\right)_{i \in \mathcal{M}}$. Let $\mathcal{E}_{\mathcal{M}}(\mathcal{P}, \mathcal{T})$ be the event that the following two statements are true for all $i \in \mathcal{M}$ :

a. $\boldsymbol{\Phi}_{i}$ has exactly $l$ positive literals.

b. For all $j \in P_{i}$ we have $\boldsymbol{\Phi}_{i j}=z_{t_{i}(j)}$.

Let $\mathcal{E}_{\mathcal{M}}$ be the event that for all $i \in \mathcal{M}$ clause $\boldsymbol{\Phi}_{i}$ has exactly $l$ positive literals among which $l-1$ are in $Z_{\theta}$. If $\mathcal{E}_{\mathcal{M}}$ occurs, then there are $\mathcal{P}, \mathcal{T}$ such that the event $\mathcal{E}_{\mathcal{M}}(\mathcal{P}, \mathcal{T})$ occurs.

For $i \in \mathcal{M}$ we let $Y_{i}=1$ if clause $\boldsymbol{\Phi}_{i}$ has exactly $l$ positive literals, including the literals $\boldsymbol{\Phi}_{i j}$ for $j \in P_{i}$. Set $\mathcal{I}=\left\{(s, i, j): i \in \mathcal{M}, j \in P_{i}, s=t_{i}(j)\right\}$. If $\mathcal{E}_{\mathcal{M}}(\mathcal{P}, \mathcal{T})$ occurs, then $\prod_{(s, i, j) \in \mathcal{I}} \mathcal{H}_{s i j} \cdot \prod_{i \in \mathcal{M}} Y_{i}=1$. Bounding $\mathrm{E}\left[\prod_{i \in \mathcal{M}} Y_{i}\right]$ as in the proof of Lemma4.9 and applying Lemma 4.8 , we obtain

$$
\mathrm{P}\left[\mathcal{E}_{\mathcal{M}}(\mathcal{P}, \mathcal{T})\right] \leq \mathrm{E}\left[\prod_{i \in \mathcal{M}} Y_{i}\right] \cdot \mathrm{E}\left[\prod_{(s, i, j) \in \mathcal{I}} \mathcal{H}_{s i j} \mid \mathcal{F}_{0}\right] \leq\left[\frac{k-l+1}{2^{k}} \cdot(n-\theta)^{1-l}\right]^{\mu} .
$$

Hence, by the union bound

$$
\begin{aligned}
\mathrm{P}\left[\mathcal{E}_{\mathcal{M}}\right] & \leq \mathrm{P}\left[\exists \mathcal{P}, \mathcal{T}: \mathcal{E}_{\mathcal{M}}(\mathcal{P}, \mathcal{T}) \text { occurs }\right] \leq \sum_{\mathcal{P}, \mathcal{T}} \mathrm{P}\left[\mathcal{E}_{\mathcal{M}}(\mathcal{P}, \mathcal{T})\right] \leq \lambda^{\mu}, \quad \text { where } \\
\lambda & =\left(\begin{array}{c}
k \\
l-1
\end{array}\right) \theta^{l-1} \times \frac{k-l+1}{2^{k}} \cdot(n-\theta)^{1-l} .
\end{aligned}
$$

Lemma2.2 implies that w.h.p. there are at most $2 \lambda m$ indices $i \in[m]$ such that $\boldsymbol{\Phi}_{i}$ has exactly $l$ positive literals of which $l-1$ lie in $Z_{\theta}$. Thus, the estimate

$$
\begin{aligned}
2 \lambda m & \leq \frac{2^{k+1} \omega n}{k} \times\left(\begin{array}{c}
k \\
l-1
\end{array}\right) \cdot \frac{k-l+1}{2^{k}} \cdot\left(\frac{\theta}{n-\theta}\right)^{l-1} & \\
& \leq 2 \omega n \cdot\left(\frac{\mathrm{e} k \theta}{(l-1)(n-\theta)}\right)^{l-1} \leq 2 \omega n\left(\frac{12 \ln \omega}{l}\right)^{l-1} \quad & \quad\left[\text { as } \theta=4 n k^{-1} \ln \omega\right] \\
& \leq n \exp (-l) & \quad[\text { because } l \geq \ln k]
\end{aligned}
$$

completes the proof.

Proof of Lemma 4.5 Since $T \leq \theta$ w.h.p. by Corollary 4.4, it suffices to show that w.h.p. for all $0 \leq t \leq$ $\min \{T, \theta\}$ the bound $\left|\mathcal{U}_{t}\right| \leq(1+\varepsilon / 3) \omega n$ holds. Let $\mathcal{U}_{t l}$ be the number of indices $i \in \mathcal{U}_{t}$ such that $\boldsymbol{\Phi}_{i}$ has precisely $l$ positive literals. Then by Lemmas 4.10 and 4.9 w.h.p. for all $t \leq \min \{T, \theta\}$ and all $1 \leq l \leq k$ simultaneously

$$
\mathcal{U}_{t l} \leq \begin{cases}n \exp (-k) & \text { if } l \geq \sqrt{k} \\ (1+\varepsilon / 9) \Lambda_{l}(t) & \text { otherwise }\end{cases}
$$

Therefore, w.h.p.

$$
\begin{aligned}
\max _{0 \leq t \leq \min \{T, \theta\}}\left|\mathcal{U}_{t}\right| & \leq \max _{0 \leq t \leq \min \{T, \theta\}} \sum_{l=1}^{k} \mathcal{U}_{t l} \leq n k \exp (-k)+\max _{0 \leq t \leq \min \{T, \theta\}} \sum_{1 \leq l \leq \sqrt{k}}^{k}(1+\varepsilon / 9) \Lambda_{l}(t) \\
& \leq n+(1+\varepsilon / 9) \omega n \leq(1+\varepsilon / 3) \omega n,
\end{aligned}
$$

as desired. 


\subsection{Proof of Corollary 4.6}

Define a map $\psi_{t}: \mathcal{U}_{t} \rightarrow V$ as follows. For $i \in \mathcal{U}_{t}$ let $s$ be the least index such $i \in \mathcal{U}_{s}$; if there is $j$ such that $\boldsymbol{\Phi}_{i j} \in V \backslash Z_{s}$, let $\psi_{t}(i)=\boldsymbol{\Phi}_{i j}$, and otherwise let $\psi_{t}(i)=z_{s}$. Thus, if $i \in U_{s}$ then $\psi_{s}(i)$ is the unique positive literal of $\boldsymbol{\Phi}_{i}$ that does not belong to $Z_{s}$. The following lemma shows that the (random) map $\psi_{t}$ is not too far from being "uniformly distributed".

Lemma 4.11 Let $t \geq 0, \hat{\mathcal{U}}_{t} \subset[m]$, and $\hat{\psi}_{t}: \hat{\mathcal{U}}_{t} \rightarrow V$. Then $\mathrm{P}\left[\psi_{t}=\hat{\psi}_{t} \mid \mathcal{U}_{t}=\hat{\mathcal{U}}_{t}\right] \leq(n-t)^{-\left|\hat{\mathcal{U}}_{t}\right|}$.

Proof. Set $Z_{-1}=\emptyset$. Moreover, define random variables

$$
\gamma_{t}(i, j)=\left\{\begin{array}{cl}
\pi_{t}(i, j) & \text { if } \pi_{t}(i, j) \in\{-1,1\} \\
0 & \text { otherwise }
\end{array} \quad \text { for }(i, j) \in[m] \times[k] .\right.
$$

Thus, $\gamma_{t}$ is obtained by "forgetting" the literals $\pi_{t}(i, j) \in V \cup \bar{V}$ that the process PI1-PI4 has revealed up to time $t$. Observe that for any $i \in[m]$

$$
i \in \mathcal{U}_{t} \Leftrightarrow \max _{j \in[k]} \gamma_{0}(i, j) \geq 0 \wedge\left(\forall j \in[k]: \gamma_{t}(i, j)=\min \left\{\gamma_{0}(i, j), 0\right\}\right)
$$

Fix a set $\hat{\mathcal{U}}_{t} \subset[m]$, let $\Phi$ be any formula such that $\mathcal{U}_{t}[\Phi]=\hat{\mathcal{U}}_{t}$, and let $\hat{\gamma}_{t}=\gamma_{t}[\Phi]$. For $s \leq t$ let $\Gamma_{s}$ be the event that $\gamma_{u}=\hat{\gamma}_{u}$ for all $u \leq s$. The goal is to prove that

$$
\mathrm{P}\left[\psi_{t}=\hat{\psi}_{t} \mid \Gamma_{t}\right] \leq(n-t)^{-\left|\hat{\mathcal{U}}_{t}\right|}
$$

Let $\tau: \hat{\mathcal{U}}_{t} \rightarrow[0, t]$ assign to each $i \in \hat{\mathcal{U}}_{t}$ the least $s$ such that $i \in \hat{\mathcal{U}}_{s}$. We claim that

$$
\mathrm{P}\left[\forall i \in \hat{\mathcal{U}}_{t}: \psi_{t}(i)=\hat{\psi}_{t}(i) \mid \Gamma_{t}\right] \leq \prod_{i \in \hat{\mathcal{U}}_{t}}(n-\tau(i))^{-1} .
$$

Since $\tau(i) \leq t$ for all $i \in \hat{\mathcal{U}}_{t}$, 23) implies (22).

Let $\tau_{s}$ be the event that $\psi_{u}(i)=\hat{\psi}_{t}(i)$ for all $0 \leq u \leq s$ and all $i \in \tau^{-1}(u)$, and let $\tau_{-1}=\Omega_{k}(n, m)$. In order to prove (23), we will show that for all $0 \leq s \leq t$

$$
\begin{aligned}
& \mathrm{P}\left[\tau_{s} \mid \tau_{s-1} \cap \Gamma_{s}\right] \leq(n-s)^{-\left|\tau^{-1}(s)\right|} \\
& \mathrm{P}\left[\tau_{s} \mid \tau_{s-1} \cap \Gamma_{s}\right]=\mathrm{P}\left[\tau_{s} \mid \tau_{s-1} \cap \Gamma_{t}\right]
\end{aligned}
$$

Combining (24) and (25) yields

$$
\begin{aligned}
\mathrm{P}\left[\forall i \in \hat{\mathcal{U}}_{t}: \psi_{t}(i)=\hat{\psi}_{t}(i) \mid \Gamma_{t}\right] & =\mathrm{P}\left[\tau_{t} \mid \Gamma_{t}\right]=\prod_{0 \leq s \leq t} \mathrm{P}\left[\tau_{s} \mid \tau_{s-1} \cap \Gamma_{t}\right] \\
& =\prod_{0 \leq s \leq t} \mathrm{P}\left[\tau_{s} \mid \tau_{s-1} \cap \Gamma_{s}\right] \leq \prod_{0 \leq s \leq t}(n-s)^{-\left|\tau^{-1}(s)\right|},
\end{aligned}
$$

which shows (23). Thus, the remaining task is to establish (24) and (25).

To prove (24) it suffices to show that

$$
\frac{\mathrm{P}\left[\tau_{s} \cap \Gamma_{s} \mid \mathcal{F}_{s-1}\right](\varphi)}{\mathrm{P}\left[\tau_{s-1} \cap \Gamma_{s} \mid \mathcal{F}_{s-1}\right](\varphi)} \leq(n-s)^{-\left|\tau^{-1}(s)\right|} \quad \text { for all } \varphi \in \tau_{s-1} \cap \Gamma_{s} .
$$

Note that the 1.h.s. is just the conditional probability of $\tau_{s}$ given $\tau_{s-1} \cap \Gamma_{s}$ with respect to the probability measure $\mathrm{P}\left[\cdot \mid \mathcal{F}_{s-1}\right](\varphi)$. Thus, let us condition on the event $\boldsymbol{\Phi} \equiv_{s-1} \varphi \in \tau_{s-1} \cap \Gamma_{s}$. Then $\boldsymbol{\Phi} \in \Gamma_{s}$, and therefore $\gamma_{0}=\hat{\gamma}_{0}$ and $\gamma_{s}=\hat{\gamma}_{s}$. Hence, 21) entails $\mathcal{U}_{s}=\mathcal{U}_{s}[\varphi]=\mathcal{U}_{s}[\Phi]$, and thus $\tau^{-1}(s) \subset \mathcal{U}_{s}$. Let $i \in \tau^{-1}(s)$, and let $J_{i}$ be the set of indices $j \in[k]$ such that $\gamma_{s-1}(i, j)=1$. Recall that $\psi_{s}(i)$ is defined as follows: if $\boldsymbol{\Phi}_{i j}=z_{s}$ for all $j \in J_{i}$, then $\psi_{s}(i)=z_{s}$; otherwise $\psi_{s}(i)=\boldsymbol{\Phi}_{i j}$ for the (unique) $j \in J_{i}$ such that $\boldsymbol{\Phi}_{i j} \neq z_{s}$. By Proposition 4.2 in the measure $\mathrm{P}\left[\cdot \mid \mathcal{F}_{s-1}\right](\varphi)$ the variables $\left(\boldsymbol{\Phi}_{i j}\right)_{i \in \tau^{-1}(s), j \in J_{i}}$ are 
independently uniformly distributed over $V \backslash Z_{s-1}$ (because $\left.\pi_{s-1}(i, j)=\gamma_{s-1}(i, j)=1\right)$. Hence, the events $\psi_{s}(i)=\hat{\psi}_{t}(i)$ occur independently for all $i \in \tau^{-1}(s)$. Thus, letting

$$
\begin{aligned}
p_{i} & =\mathrm{P}\left[\psi_{s}(i)=\psi_{t}(i) \wedge \forall j \in J_{i}: \gamma_{s}(i, j)=0 \mid \mathcal{F}_{s-1}\right](\varphi) \\
q_{i} & =\mathrm{P}\left[\forall j \in J_{i}: \gamma_{s}(i, j)=0 \mid \mathcal{F}_{s-1}\right](\varphi)
\end{aligned}
$$

for $i \in \tau^{-1}(s)$, we have

$$
\frac{\mathrm{P}\left[\tau_{s} \cap \Gamma_{s} \mid \mathcal{F}_{s-1}\right](\varphi)}{\mathrm{P}\left[\tau_{s-1} \cap \Gamma_{s} \mid \mathcal{F}_{s-1}\right](\varphi)}=\prod_{i \in \tau^{-1}(s)} \frac{p_{i}}{q_{i}}
$$

Observe that the event $\forall j \in J_{i}: \gamma_{s}(i, j)=0$ occurs iff $\boldsymbol{\Phi}_{i j}=z_{s}$ for at least $\left|J_{i}\right|-1$ elements $j \in J_{i}$ (cf. PI4). Therefore,

$$
q_{i}=\left|J_{i}\right| \cdot\left|V \backslash Z_{s-1}\right|^{-\left(\left|J_{i}\right|-1\right)}\left(1-\left|V \backslash Z_{s-1}\right|^{-1}\right)+\left|V \backslash Z_{s-1}\right|^{-\left|J_{i}\right|}
$$

To bound $p_{i}$ for $i \in \tau^{-1}(s)$ we consider three cases.

Case 1: $\hat{\psi}_{t}(i) \in V \backslash Z_{s-1}$. As $\boldsymbol{\Phi}_{i j} \in V \backslash Z_{s-1}$ for all $j \in J_{i}$ the event $\psi_{s}(i)=\hat{\psi}_{t}(i)$ has probability 0 .

Case 2: $\hat{\psi}_{t}(i)=z_{s}$. The event $\psi_{s}(i)=\hat{\psi}_{t}(i)$ occurs iff $\boldsymbol{\Phi}_{i j}=z_{s}$ for all $j \in J_{i}$, which happens with probability $\left|V \backslash Z_{s-1}\right|^{-\left|J_{i}\right|}$ in the measure $\mathrm{P}\left[\cdot \mid \mathcal{F}_{s-1}\right](\varphi)$. Hence, $p_{i}=(n-s+1)^{-\left|J_{i}\right|}$.

Case 3: $\hat{\psi}_{t}(i) \in V \backslash Z_{s}$. If $\psi_{s}(i)=\hat{\psi}_{t}(i)$, then there is $j \in J_{i}$ such that $\boldsymbol{\Phi}_{i j}=\hat{\psi}_{t}(i)$ and $\boldsymbol{\Phi}_{i j^{\prime}}=z_{s}$ for all $j^{\prime} \in J_{s} \backslash\{j\}$. Hence, $p_{i}=\left|J_{i}\right| \cdot\left|V \backslash Z_{s-1}\right|^{-\left|J_{i}\right|}=\left|J_{i}\right|(n-s+1)^{-\left|J_{i}\right|}$.

In all three cases we have

$$
\frac{q_{i}}{p_{i}} \geq \frac{\left|J_{i}\right|(n-s+1)^{1-\left|J_{i}\right|}(1-1 /(n-s+1))}{\left|J_{i}\right|(n-s+1)^{-\left|J_{i}\right|}}=n-s .
$$

Thus, 26) follows from 27).

In order to prove 25] we will show that

$$
\mathrm{P}\left[\Gamma_{a} \mid \tau_{b} \cap \Gamma_{c}\right]=\mathrm{P}\left[\Gamma_{a} \mid \Gamma_{c}\right]
$$

for any $0 \leq b \leq c<a$. This implies 25] as follows:

$$
\begin{aligned}
\mathrm{P}\left[\tau_{s} \mid \tau_{s-1} \cap \Gamma_{t}\right] & =\frac{\mathrm{P}\left[\tau_{s} \cap \Gamma_{t}\right]}{\mathrm{P}\left[\tau_{s-1} \cap \Gamma_{t}\right]}=\frac{\mathrm{P}\left[\Gamma_{t} \mid \tau_{s} \cap \Gamma_{s}\right] \mathrm{P}\left[\tau_{s} \cap \Gamma_{s}\right]}{\mathrm{P}\left[\Gamma_{t} \mid \tau_{s-1} \cap \Gamma_{s}\right] \mathrm{P}\left[\tau_{s-1} \cap \Gamma_{s}\right]} \\
& \stackrel{\mathrm{288}}{=} \frac{\mathrm{P}\left[\tau_{s} \cap \Gamma_{s}\right]}{\mathrm{P}\left[\tau_{s-1} \cap \Gamma_{s}\right]}=\mathrm{P}\left[\tau_{s} \mid \tau_{s-1} \cap \Gamma_{s}\right]
\end{aligned}
$$

To show (28) it suffices to consider the case $a=c+1$, because for $a>c+1$ we have

$$
\begin{aligned}
\mathrm{P}\left[\Gamma_{a} \mid \tau_{b} \cap \Gamma_{c}\right] & =\mathrm{P}\left[\Gamma_{a} \mid \tau_{b} \cap \Gamma_{c+1}\right] \mathrm{P}\left[\tau_{b} \cap \Gamma_{c+1} \mid \tau_{b} \cap \Gamma_{c}\right] \\
& =\mathrm{P}\left[\Gamma_{a} \mid \tau_{b} \cap \Gamma_{c+1}\right] \mathrm{P}\left[\Gamma_{c+1} \mid \tau_{b} \cap \Gamma_{c}\right] .
\end{aligned}
$$

Thus, suppose that $a=c+1$. At time $a=c+1$ PI1 selects an index $\phi_{a} \in[m]$. This is the least index $i$ such that $\gamma_{c}(i, j)=-1$ for all $j$; thus, $\phi_{a}$ is determined once we condition on $\Gamma_{c}$. Then, PI2 selects a variable $z_{a}=\left|\boldsymbol{\Phi}_{\phi_{a} j_{a}}\right|$ with $j_{a} \leq k_{1}$. Now, $\gamma_{a}$ is obtained from $\gamma_{c}$ by setting the entries for some $(i, j)$ such that $\gamma_{c}(i, j) \in\{-1,1\}$ to 0 (cf. PI4). More precisely, we have $\gamma_{a}\left(\phi_{a}, j\right)=0$ for all $j \leq k_{1}$. Furthermore, for $i \in[m] \backslash\left\{\phi_{a}\right\}$ let $\mathcal{J}_{i}$ be the set of all $j \in[k]$ such that $\pi_{a}(i, j)=\gamma_{a}(i, j) \in\{-1,1\}$, and for $i=\phi_{a}$ let $\mathcal{J}_{i}$ be the set of all $k_{1}<j \leq k$ such that $\pi_{a}(i, j)=\gamma_{a}(i, j) \in\{-1,1\}$. Then for any $i \in[m]$ and any $j \in \mathcal{J}_{i}$ the event $\gamma_{c}(i, j)=0$ only depends on the events $\left|\boldsymbol{\Phi}_{i j^{\prime}}\right|=z_{a}$ for $j^{\prime} \in \mathcal{J}_{i}$. By Proposition 4.2 the variables $\left(\left|\boldsymbol{\Phi}_{i j^{\prime}}\right|\right)_{i \in[m], j \in \mathcal{J}_{i}}$ are independently uniformly distributed over $V \backslash Z_{c}$. Therefore, the events $\left|\boldsymbol{\Phi}_{i j^{\prime}}\right|=z_{a}$ for $j^{\prime} \in \mathcal{J}_{i}$ are independent of the choice of $z_{a}$ and of the event $\tau_{b}$. 
Proof of Corollary 4.6] Let $\mu \leq(1+\varepsilon / 3) \omega n$ be a positive integer and let $\hat{\mathcal{U}}_{t} \subset[m]$ be a set of size $\mu$. Suppose that $t \leq \theta$. Let $\nu=n k^{-\varepsilon / 2}$, and let $B$ be the set of all maps $\psi: \hat{\mathcal{U}}_{t} \rightarrow[n]$ such that there are less than $\nu+t$ numbers $x \in[n]$ such that $\psi^{-1}(x)=\emptyset$. Furthermore, let $\mathcal{B}_{t}$ be the event that there are less than $\nu$ variables $x \in V \backslash Z_{t}$ such that $\mathcal{U}_{t}(x)=0$. Since $\left|Z_{t}\right|=t$, we have

$$
\begin{aligned}
\mathrm{P}\left[\mathcal{B}_{t} \mid \mathcal{U}_{t}=\hat{\mathcal{U}}_{t}\right] & \leq \sum_{\psi \in B} \mathrm{P}\left[\psi_{t}=\psi \mid \mathcal{U}_{t}=\hat{\mathcal{U}}_{t}\right] \leq|B|(n-t)^{-\mu} \quad \text { [by Lemma 4.11] } \\
& =\frac{|B|}{n^{\mu}} \cdot\left(1+\frac{t}{n-t}\right)^{\mu} \leq \frac{|B|}{n^{\mu}} \cdot \exp (2 \theta \mu / n) \leq \frac{|B|}{n^{\mu}} \cdot \exp \left(9 n k^{-1} \ln ^{2} k\right) .
\end{aligned}
$$

Furthermore, $|B| / n^{\mu}$ is just the probability that there are less than $\nu$ empty bins if $\mu$ balls are thrown uniformly and independently into $n$ bins. Hence, we can use Lemma 2.1 to bound $|B| n^{-\mu}$. To this end, observe that because we are assuming $\varepsilon<0.1$ the bound

$$
\exp (-\mu / n) \geq \exp (-(1+\varepsilon / 3) \omega)=k^{\alpha-1} \quad \text { holds, where } \alpha=\frac{2 \varepsilon}{3}-\frac{\varepsilon^{2}}{3} \geq 0.6 \varepsilon .
$$

Therefore, Lemma2.1 entails that

$$
\begin{aligned}
|B| n^{-\mu} & \leq \mathrm{P}[\mathcal{Z}(\mu, n) \leq \exp (-\mu / n) n / 2] \\
& \leq O(\sqrt{n}) \exp [-\exp (-\mu / n) n / 8] \leq \exp \left[-k^{\alpha-1} n / 9\right] .
\end{aligned}
$$

Combining (29) and (30), we see that

$$
P_{t}=\mathrm{P}\left[\mathcal{B}_{t}\left|\mathcal{U}_{t}=\hat{\mathcal{U}}_{t}: \hat{\mathcal{U}}_{t} \subset[m],\right| \hat{\mathcal{U}}_{t} \mid=\mu\right] \leq \exp \left[n k^{-1}\left(9 \ln ^{2} k-k^{\alpha} / 9\right)\right]=o(1 / n) .
$$

Thus, Corollary 4.4 and Lemma4.5imply that

$$
\begin{aligned}
\mathrm{P}[\exists t \leq T: \mid\{x \in & \left.\left.V \backslash Z_{t}: \mathcal{U}_{t}(x)=0\right\}<\nu \mid\right] \\
& \leq \mathrm{P}[T>\theta]+\mathrm{P}\left[\max _{0 \leq t \leq T}\left|\mathcal{U}_{t}\right|>(1+\varepsilon / 3) \omega n\right]+\sum_{0 \leq t \leq \theta} P_{t}=o(1),
\end{aligned}
$$

as desired.

\section{Proof of Proposition 3.3}

Let $0<\varepsilon<0.1$. Throughout this section we assume that $k \geq k_{0}$ for a large enough $k_{0}=k_{0}(\varepsilon)$, and that $n>n_{0}$ for some large enough $n_{0}=n_{0}(\varepsilon, k)$. Let $m=\left\lfloor(1-\varepsilon) 2^{k} k^{-1} \ln k\right\rfloor, \omega=(1-\varepsilon) \ln k$, and $k_{1}=\lceil k / 2\rceil$. In addition, we keep the notation introduced in Section 4.1

\subsection{Outline}

Similarly as in Section 4 we will describe the execution of Phase 2 of Fix $(\Phi)$ via a stochastic process. Recall that $T$ denotes the time when the process PI1-PI4 from Section4(i.e., Phase 1) stops. Let $Z_{0}^{\prime}=\emptyset$ and $\pi_{0}^{\prime}=\pi_{T}$. Let $U_{0}^{\prime}=U_{T}$, and let $U_{0}^{\prime}(x)$ be the number of indices $i \in U_{0}^{\prime}$ such that $x$ occurs positively in $\boldsymbol{\Phi}_{i}$. Moreover, let $Q_{0}^{\prime}$ be the set of indices $i \in[m]$ such that $\boldsymbol{\Phi}_{i}$ is unsatisfied under $\sigma_{Z_{T}}$. For $t \geq 1$ we proceed as follows.

PI1' If $Q_{t-1}^{\prime}=\emptyset$, the process stops. Otherwise let $\psi_{t}=\min Q_{t-1}^{\prime}$.

PI2' If there are three indices $k_{1}<j \leq k-5$ such that $\pi_{t-1}^{\prime}\left(\psi_{t}, j\right) \in\{1,-1\}$ and $U_{t-1}^{\prime}\left(\left|\Phi_{\psi_{t} j}\right|\right)=0$, then let $k_{1}<j_{1}<j_{2}<j_{3} \leq k-5$ be the lexicographically first sequence of such indices. Otherwise let $k-5<j_{1}<j_{2}<j_{3} \leq k$ be the lexicographically first sequence of indices $k-5<j \leq k$ such that $\Phi_{\psi_{t} j} \notin Z_{t-1}^{\prime}$. Let $Z_{t}^{\prime}=Z_{t-1}^{\prime} \cup\left\{\left|\boldsymbol{\Phi}_{\psi_{t} j_{l}}\right|: l=1,2,3\right\}$. 
PI3' Let $U_{t}^{\prime}$ be the set of all $i \in[m]$ that satisfy the following condition. There is exactly one $l \in[k]$ such that $\boldsymbol{\Phi}_{i l} \in V \backslash\left(Z_{t}^{\prime} \cup Z_{T}\right)$ and for all $j \neq l$ we have $\boldsymbol{\Phi}_{i j} \in Z_{T} \cup Z_{t}^{\prime} \cup \overline{V \backslash Z_{T}}$. Let $U_{t}^{\prime}(x)$ be the number of indices $i \in U_{t}^{\prime}$ such that $x$ occurs positively in $\Phi_{i}(x \in V)$.

PI4' Let

$$
\pi_{t}^{\prime}(i, j)=\left\{\begin{array}{cl}
\boldsymbol{\Phi}_{i j} & \text { if }\left(i=\psi_{t} \wedge j>k_{1}\right) \vee\left|\boldsymbol{\Phi}_{i j}\right| \in Z_{t}^{\prime} \cup Z_{T} \vee\left(i \in U_{t}^{\prime} \wedge \pi_{0}(i, j)=1\right), \\
\pi_{t-1}^{\prime}(i, j) & \text { otherwise. }
\end{array}\right.
$$

Let $Q_{t}^{\prime}$ be the set of all $\left(Z_{T}, Z_{t}^{\prime}\right)$-endangered clauses that contain less than three variables from $Z_{t}^{\prime}$.

Let $T^{\prime}$ be the stopping time of this process. For $t>T^{\prime}$ and $x \in V$ let $\pi_{t}^{\prime}=\pi_{T^{\prime}}^{\prime}, U_{t}^{\prime}=U_{T^{\prime}}^{\prime}, Z_{t}^{\prime}=Z_{T^{\prime}}^{\prime}$, and $U_{t}^{\prime}(x)=U_{T^{\prime}}(x)$.

We define an equivalence relation $\equiv_{t}^{\prime}$ by letting $\Phi \equiv_{t}^{\prime} \Psi$ iff $\Phi \equiv_{s} \Psi$ for all $s \geq 0$, and $\pi_{s}^{\prime}[\Phi]=\pi_{s}^{\prime}[\Psi]$ for all $0 \leq s \leq t$. Let $\mathcal{F}_{t}^{\prime}$ be the $\sigma$-algebra generated by the equivalence classes of $\equiv_{t}^{\prime}$. Then $\left(\mathcal{F}_{t}^{\prime}\right)_{t \geq 0}$ is a filtration.

Fact 5.1 For any $t \geq 0$ the map $\pi_{t}^{\prime}$, the random variable $\psi_{t+1}^{\prime}$, the random sets $U_{t}^{\prime}$ and $Z_{t}^{\prime}$, and the random variables $U_{t}^{\prime}(x)$ for $x \in V$ are $\mathcal{F}_{t}^{\prime}$-measurable.

The same argument that we used to prove Proposition 4.2 in Section 4.1 shows the following.

Proposition 5.2 Let $\mathcal{E}_{t}^{\prime}$ be the set of all pairs $(i, j)$ such that $\pi_{t}(i, j) \in\{ \pm 1\}$. The conditional joint distribution of the variables $\left(\left|\Phi_{i j}\right|\right)_{(i, j) \in \mathcal{E}_{t}}$ given $\mathcal{F}_{t}^{\prime}$ is uniform over $\left(V \backslash Z_{t}^{\prime}\right)^{\mathcal{E}_{t}^{\prime}}$.

Let

$$
\theta^{\prime}=\left\lfloor\exp \left(-k^{\varepsilon / 16}\right) n\right\rfloor, \text { and recall that } \theta=\left\lfloor 4 n k^{-1} \ln \omega\right\rfloor .
$$

To prove Proposition 3.3 it is sufficient to show that $T^{\prime} \leq \theta^{\prime}$ w.h.p., because $\left|Z_{t}^{\prime}\right|=3 t$ for all $t \leq T^{\prime}$. To this end, we follow a similar program as in Section 4.1; we will show that $\left|U_{t}^{\prime}\right|$ is "small" w.h.p. for all $t \leq \theta^{\prime}$, and that therefore for $t \leq \theta^{\prime}$ there are plenty of variables $x$ such that $U_{t}^{\prime}(x)=0$. This implies that for $t \leq \theta^{\prime}$ the process will only "generate" very few $\left(Z_{T}, Z_{t}^{\prime}\right)$-endangered clauses. This then entails a bound on $\bar{T}^{\prime}$, because each step of the process removes (at least) one $\left(Z_{T}, Z_{t}^{\prime}\right)$-endangered clause from the set $Q_{t}^{\prime}$. In Section 5.2 we will infer the following bound on $\left|U_{t}^{\prime}\right|$.

Lemma 5.3 W.h.p. for all $t \leq \theta^{\prime}$ we have $\left|U_{t}^{\prime} \backslash U_{T}\right| \leq n / k$.

Corollary 5.4 W.h.p. the following is true for all $t \leq \theta^{\prime}$ : there are at least $n k^{\varepsilon / 3-1}$ variables $x \in V \backslash$ $\left(Z_{t}^{\prime} \cup Z_{T}\right)$ such that $U_{t}^{\prime}(x)=0$.

Proof. By Corollary 4.6 there are at least $n k^{\varepsilon / 2-1}$ variables $x \in V \backslash Z_{T}$ such that $U_{T}(x)=0$ w.h.p. Furthermore, by Lemma 5.3 we have $\left|U_{t}^{\prime} \backslash U_{T}\right| \leq n / k$ w.h.p. Moreover, $\left|Z_{t}^{\prime}\right| \leq 3 t$. Hence, w.h.p. the number of $x \in V \backslash\left(Z_{t}^{\prime} \cup Z_{T}\right)$ such that $U_{t}^{\prime}(x)=0$ is at least $n k^{\varepsilon / 2-1}-n / k-3 \theta^{\prime} \geq n k^{\varepsilon / 3-1}$.

Corollary 5.5 Let $\mathcal{Y}$ be the set of all $t \leq \theta^{\prime}$ such that there are less than 3 indices $k_{1}<j \leq k-5$ such that $\pi_{t-1}^{\prime}\left(\psi_{t}, j\right) \in\{-1,1\}$ and $U_{t-1}^{\prime}\left(\left|\Phi_{\psi_{t} j}\right|\right)=0$. Then $|\mathcal{Y}| \leq 3 \theta^{\prime} \exp \left(-k^{\varepsilon / 4}\right)$ w.h.p.

We defer the proof of Corollary 5.5 to Section 5.3. Furthermore, in Section 5.4 we will prove the following.

Corollary 5.6 W.h.p. the total number of $\left(Z_{T}, Z_{\theta^{\prime}}^{\prime}\right)$-endangered clauses is at most $\theta^{\prime}$.

Proof of Proposition 3.3 We claim that $T^{\prime} \leq \theta^{\prime}$ w.h.p.; this implies the proposition because $\left|Z_{T^{\prime}}\right|=3 T^{\prime}$. To see that $T^{\prime} \leq \theta^{\prime}$ w.h.p., let $X_{0}$ be the total number of $\left(Z_{T}, Z_{\theta^{\prime}}^{\prime}\right)$-endangered clauses, and let $X_{t}$ be the number of $\left(Z_{T}, Z_{\theta^{\prime}}^{\prime}\right)$-endangered clauses that contain less than 3 variables from $Z_{t}^{\prime}$. Then the construction PI1'-PI4' ensures that $0 \leq X_{t} \leq X_{0}-t$ for all $t \leq T^{\prime}$. Hence, $T^{\prime} \leq X_{0}$, and thus the assertion follows from Corollary 5.6 . 


\subsection{Proof of Lemma 5.3}

Let $\mathcal{H}_{t i j}, \mathcal{S}_{t i j}$ be as in 14 and let in addition

$$
\mathcal{H}_{t i j}^{\prime}= \begin{cases}1 & \text { if } \pi_{t-1}^{\prime}(i, j)=1, \pi_{t}^{\prime}(i, j) \in Z_{t}^{\prime}, \text { and } T \leq \theta \\ 0 & \text { otherwise }\end{cases}
$$

Lemma 5.7 For any $\mathcal{I}^{\prime} \subset\left[\theta^{\prime}\right] \times[m] \times[k]$ we have $\mathrm{E}\left[\prod_{(t, i, j) \in \mathcal{I}^{\prime}} \mathcal{H}_{t i j}^{\prime} \mid \mathcal{F}_{0}^{\prime}\right] \leq\left(3 /\left(n-\theta-3 \theta^{\prime}\right)\right)^{\left|\mathcal{I}^{\prime}\right|}$.

Proof. Let $\mathcal{I}_{t}^{\prime}=\left\{(i, j):(t, i, j) \in \mathcal{I}^{\prime}\right\}$ and $X_{t}=\prod_{(i, j) \in \mathcal{I}_{t}^{\prime}} \mathcal{H}_{t i j}^{\prime}$. Due to Lemma2.4 it suffices to show

$$
\mathrm{E}\left[X_{t} \mid \mathcal{F}_{t-1}^{\prime}\right] \leq\left(3 /\left(n-\theta-3 \theta^{\prime}\right)\right)^{\left|\mathcal{I}_{t}^{\prime}\right|} \quad \text { for all } t \leq \theta^{\prime}
$$

To see this, let $1 \leq t \leq \theta^{\prime}$ and consider a formula $\Phi$ such that $T[\Phi] \leq \theta, t \leq T^{\prime}[\Phi]$, and $\pi_{t-1}^{\prime}(i, j)[\Phi]=1$ for all $(i, j) \in \mathcal{I}_{t}^{\prime}$. We condition on the event $\boldsymbol{\Phi} \equiv_{t-1}^{\prime} \Phi$. Then at time $t$ steps PI1'-PI2' obtain $Z_{t}^{\prime}$ by adding three variables that occur in clause $\boldsymbol{\Phi}_{\psi_{t}}$, which is $\left(Z_{T}, Z_{t-1}^{\prime}\right)$-endangered. Let $(i, j) \in \mathcal{I}_{t}^{\prime}$. Since $\boldsymbol{\Phi} \equiv_{t-1} \Phi$ and $\pi_{t-1}(i, j)[\Phi]=1$, the literal $\boldsymbol{\Phi}_{i j} \notin Z_{T} \cup Z_{t-1}^{\prime}$ is positive, and thus $\boldsymbol{\Phi}_{i}$ is not $\left(Z_{T}, Z_{t-1}^{\prime}\right)$ endangered. Hence, $\psi_{t} \neq i$. Furthermore, by Proposition 5.2 in the conditional distribution $\mathrm{P}\left[\cdot \mid \mathcal{F}_{t-1}^{\prime}\right](\Phi)$ the variables $\left(\boldsymbol{\Phi}_{i j}\right)_{(i, j) \in \mathcal{I}_{t}^{\prime}}$ are independently uniformly distributed over the set $V \backslash\left(Z_{T} \cup Z_{t-1}^{\prime}\right)$. Hence,

$$
\mathrm{P}\left[\boldsymbol{\Phi}_{i j} \in Z_{t}^{\prime} \mid \mathcal{F}_{t-1}^{\prime}\right][\Phi]=3 /\left|V \backslash\left(Z_{T} \cup Z_{t-1}^{\prime}\right)\right| \quad \text { for any }(i, j) \in \mathcal{I}_{t}^{\prime},
$$

and these events are mutually independent. Since $\left|Z_{T}\right|=n-T$ and $T \leq \theta$, and because $\left|Z_{t-1}^{\prime}\right|=3(t-1)$, (32) implies 31.

Lemma 5.8 Let $2 \leq l \leq \sqrt{k}, 1 \leq l^{\prime} \leq l-1,1 \leq t \leq \theta$, and $1 \leq t^{\prime} \leq \theta^{\prime}$. For each $i \in[m]$ let $X_{i}=1$ if $T \geq t, T^{\prime} \geq t^{\prime}$, and the following four events occur:

a. $\boldsymbol{\Phi}_{i}$ has exactly l positive literals.

b. $l^{\prime}$ of the positive literals of $\boldsymbol{\Phi}_{i}$ lie in $Z_{t^{\prime}}^{\prime} \backslash Z_{t}$.

c. $l-l^{\prime}-1$ of the positive literals of $\boldsymbol{\Phi}_{i}$ lie in $Z_{t}$.

d. No variable from $Z_{t}$ occurs in $\boldsymbol{\Phi}_{i}$ negatively.

Let

$$
B\left(l, l^{\prime}, t\right)=4 \omega n \cdot\left(\frac{6 \theta^{\prime} k}{n}\right)^{l^{\prime}} \cdot\left(\begin{array}{c}
k-l^{\prime}-1 \\
l-l^{\prime}-1
\end{array}\right)\left(\frac{t}{n}\right)^{l-l^{\prime}-1}(1-t / n)^{k-l} .
$$

Then $\mathrm{P}\left[\sum_{i=1}^{m} X_{i}>B\left(l, l^{\prime}, t\right)\right]=o\left(n^{-3}\right)$.

Proof. We are going to apply Lemma2.2 Set $\mu=\left\lceil\ln ^{2} n\right\rceil$ and let $\mathcal{M} \subset[m]$ be a set of size $\mu$. Let $\mathcal{E}_{\mathcal{M}}$ be the event that $X_{i}=1$ for all $i \in \mathcal{M}$. Let $P_{i} \subset[k]$ be a set of size $l$, and let $H_{i}, H_{i}^{\prime} \subset P_{i}$ be disjoint sets such that $\left|H_{i} \cup H_{i}^{\prime}\right|=l-1$ and $\left|H_{i}^{\prime}\right|=l^{\prime}$ for each $i \in \mathcal{M}$. Let $\mathcal{P}=\left(P_{i}, H_{i}, H_{i}^{\prime}\right)_{i \in \mathcal{M}}$. Furthermore, let $t_{i}: H_{i} \rightarrow[t]$ and $t_{i}^{\prime}: H_{i}^{\prime} \rightarrow\left[t^{\prime}\right]$ for all $i \in \mathcal{M}$, and set $\mathcal{T}=\left(t_{i}, t_{i}^{\prime}\right)_{i \in \mathcal{M}}$. Let $\mathcal{E}_{\mathcal{M}}(\mathcal{P}, \mathcal{T})$ be the event that $T \geq t, T^{\prime} \geq t^{\prime}$, and the following statements are true for all $i \in \mathcal{M}$ :

a'. The literal $\boldsymbol{\Phi}_{i j}$ is positive for all $j \in P_{i}$ and negative for all $j \in[k] \backslash P_{i}$.

b'. $\boldsymbol{\Phi}_{i j} \in Z_{t_{i}^{\prime}(j)}^{\prime} \backslash Z_{t_{i}^{\prime}(j)-1}^{\prime}$ for all $i \in \mathcal{M}$ and $j \in H_{i}^{\prime}$.

c'. $\boldsymbol{\Phi}_{i j}=z_{t_{i}(j)}$ for all $i \in \mathcal{M}$ and $j \in H_{i}$.

d'. No variable from $Z_{t}$ occurs negatively in $\boldsymbol{\Phi}_{i}$. 
If $\mathcal{E}_{\mathcal{M}}$ occurs, then there exist $(\mathcal{P}, \mathcal{T})$ such that $\mathcal{E}_{\mathcal{M}}(\mathcal{P}, \mathcal{T})$ occurs. Hence, we are going to use the union bound. For each $i \in[M]$ there are

$$
\left(\begin{array}{c}
k \\
1, l^{\prime}, l-l^{\prime}-1
\end{array}\right) \text { ways to choose the sets } P_{i}, H_{i}, H_{i}^{\prime} .
$$

Once these are chosen, there are

$$
t^{\prime l^{\prime}} \text { ways to choose the map } t_{i}^{\prime} \text {, and } t^{l-l^{\prime}-1} \text { ways to choose the map } t_{i} \text {. }
$$

Thus,

$$
\mathrm{P}\left[\mathcal{E}_{\mathcal{M}}\right] \leq \sum_{\mathcal{P}, \mathcal{T}} \mathrm{P}\left[\mathcal{E}_{\mathcal{M}}(\mathcal{P}, \mathcal{T})\right] \leq\left[\left(\begin{array}{c}
k \\
1, l^{\prime}, l-l^{\prime}-1
\end{array}\right) t^{t^{\prime}} t^{l-l^{\prime}-1}\right]^{\mu} \max _{\mathcal{P}, \mathcal{T}} \mathrm{P}\left[\mathcal{E}_{\mathcal{M}}(\mathcal{P}, \mathcal{T})\right]
$$

Hence, we need to bound $\mathrm{P}\left[\mathcal{E}_{\mathcal{M}}(\mathcal{P}, \mathcal{T})\right]$ for any given $\mathcal{P}, \mathcal{T}$. To this end, let

$$
\begin{aligned}
\mathcal{I} & =\mathcal{I}(\mathcal{M}, \mathcal{P}, \mathcal{T})=\left\{(s, i, j): i \in \mathcal{M}, j \in P_{i}, s=t_{i}(j)\right\} \\
\mathcal{I}^{\prime} & =\mathcal{I}^{\prime}(\mathcal{M}, \mathcal{P}, \mathcal{T})=\left\{(s, i, j): i \in \mathcal{M}, j \in P_{i}^{\prime}, s=t_{i}^{\prime}(j)\right\} \\
\mathcal{J} & =\mathcal{J}(\mathcal{M}, \mathcal{P}, \mathcal{T})=\left\{(s, i, j): i \in \mathcal{M}, j \in[k] \backslash\left(P_{i} \cup P_{i}^{\prime}\right), s \leq t\right\} .
\end{aligned}
$$

If $\mathcal{E}_{\mathcal{M}}(\mathcal{P}, \mathcal{T})$ occurs, then the positive literals of each clause $\boldsymbol{\Phi}_{i}, i \in \mathcal{M}$, are precisely $\boldsymbol{\Phi}_{i j}$ with $j \in P_{i}$, which occurs with probability $2^{-k}$ independently. In addition, we have $\mathcal{H}_{s i j}=1$ for all $(s, i, j) \in \mathcal{I}$, $\mathcal{H}_{s i j}^{\prime}=1$ for all $(s, i, j) \in \mathcal{I}^{\prime}$, and $\mathcal{S}_{s i j}=1$ for all $(s, i, j) \in \mathcal{J}$. Hence, by Lemmas 4.8 and 5.7

$$
\begin{aligned}
\mathrm{P}\left[\mathcal{E}_{\mathcal{M}}(\mathcal{P}, \mathcal{T})\right] & \leq 2^{-k \mu} \cdot \mathrm{E}\left[\prod_{(t, i, j) \in \mathcal{I}^{\prime}} \mathcal{H}_{t i j}^{\prime} \cdot \prod_{(t, i, j) \in \mathcal{I}} \mathcal{H}_{t i j} \cdot \prod_{(t, i, j) \in \mathcal{J}} \mathcal{S}_{t i j} \mid \mathcal{F}_{0}\right] \\
& \leq 2^{-k \mu} \cdot\left(\frac{3}{n-\theta-3 \theta^{\prime}}\right)^{l^{\prime} \mu}(n-\theta)^{-\left(l-l^{\prime}-1\right) \mu}(1-1 / n)^{(k-l) t \mu}
\end{aligned}
$$

Combining (33) and (34), we see that $\mathrm{P}\left[\mathcal{E}_{\mathcal{M}}\right] \leq \lambda^{\mu}$, where

$$
\lambda=2^{-k}\left(\begin{array}{c}
k \\
1, l^{\prime}, l-l^{\prime}-1
\end{array}\right)\left(\frac{3 t^{\prime}}{n-\theta-3 \theta^{\prime}}\right)^{l^{\prime}}\left(\frac{t}{n-\theta}\right)^{l-l^{\prime}-1}(1-1 / n)^{(k-l) t},
$$

whence Lemma 2.2 yields $\mathrm{P}\left[\sum_{i=1}^{m} X_{i}>2 \lambda m\right]=o\left(n^{-3}\right)$. Thus, the remaining task is to estimate $\lambda m$ :

$$
\begin{aligned}
\lambda m & =m k 2^{-k}\left(\begin{array}{c}
k-1 \\
l^{\prime}
\end{array}\right)\left(\frac{3 t^{\prime}}{n-\theta-3 \theta^{\prime}}\right)^{l^{\prime}} \cdot\left(\begin{array}{c}
k-l^{\prime}-1 \\
l-l^{\prime}-1
\end{array}\right)\left(\frac{t}{n-\theta}\right)^{l-l^{\prime}-1}(1-1 / n)^{(k-l) t} \\
& \leq \omega n \cdot\left(\frac{6 \theta^{\prime} k}{n}\right)^{l^{\prime}} \cdot\left(\begin{array}{c}
k-l^{\prime}-1 \\
l-l^{\prime}-1
\end{array}\right)\left(\frac{t}{n}\right)^{l-l^{\prime}-1}(1-t / n)^{k-l} \cdot \eta, \quad \text { where } \\
\eta & =\left(\frac{n}{n-\theta}\right)^{l-l^{\prime}-1} \cdot\left(\frac{(1-1 / n)^{t}}{1-t / n}\right)^{k-l} \\
& \leq\left(1+\frac{\theta}{n-\theta}\right)^{l-l^{\prime}-1} \exp \left(k t^{2} / n^{2}\right) \leq \exp \left(2 \theta l / n+k \theta^{2} / n^{2}\right) .
\end{aligned}
$$

Since $\theta \leq 4 k^{-1} n \ln k$ and $l \leq \sqrt{k}$, we have $\eta \leq 2$ for large $k$. Thus, the assertion follows from (35).

Lemma 5.9 Let $\ln k \leq l \leq k, 1 \leq l^{\prime} \leq l, 1 \leq t \leq \theta$, and $1 \leq t^{\prime} \leq \theta^{\prime}$. For each $i \in[m]$ let $Y_{i}=1$ if $T \geq t, T^{\prime} \geq t^{\prime}$, and the following three events occur:

a. $\boldsymbol{\Phi}_{i}$ has exactly l positive literals. 
b. $l^{\prime}$ of the positive literals of $\boldsymbol{\Phi}_{i}$ lie in $Z_{t^{\prime}}^{\prime} \backslash Z_{t}$.

c. $l-l^{\prime}-1$ of the positive literals of $\boldsymbol{\Phi}_{i}$ lie in $Z_{t}$.

Then $\mathrm{P}\left[\sum_{i=1}^{m} Y_{i}>n \exp (-l)\right]=o\left(n^{-3}\right)$.

Proof. The proof is similar to (and less involved than) the proof of Lemma 5.9 Set $\mu=\left\lceil\ln ^{2} n\right\rceil$ and let $\mathcal{M} \subset[m]$ be a set of size $\mu$. Let $\mathcal{E}_{\mathcal{M}}$ be the event that $Y_{i}=1$ for all $i \in[M]$. Let $P_{i} \subset[k]$ be a set of size $l$, and let $H_{i}, H_{i}^{\prime} \subset P_{i}$ be disjoint sets such that $\left|H_{i} \cup H_{i}^{\prime}\right|=l-1$ and $\left|H_{i}^{\prime}\right|=l^{\prime}$ for each $i \in \mathcal{M}$. Let $\mathcal{P}=\left(P_{i}, H_{i}, H_{i}^{\prime}\right)_{i \in \mathcal{M}}$. Furthermore, let $t_{i}: H_{i} \rightarrow[t]$ and $t_{i}^{\prime}: H_{i}^{\prime} \rightarrow\left[t^{\prime}\right]$ for all $i \in \mathcal{M}$, and set $\mathcal{T}=\left(t_{i}, t_{i}^{\prime}\right)_{i \in \mathcal{M}}$. Let $\mathcal{E}_{\mathcal{M}}(\mathcal{P}, \mathcal{T})$ be the event that $T \geq t, T^{\prime} \geq t^{\prime}$, and the following statements are true for all $i \in \mathcal{M}$ :

a'. $\boldsymbol{\Phi}_{i j}$ is positive for all $j \in P_{i}$ and negative for all $j \notin P_{i}$.

b'. $\boldsymbol{\Phi}_{i j} \in Z_{t_{i}^{\prime}(j)}^{\prime} \backslash Z_{t_{i}^{\prime}(j)-1}^{\prime}$ for all $i \in \mathcal{M}$ and $j \in H_{i}^{\prime}$.

c'. $\boldsymbol{\Phi}_{i j}=z_{t_{i}(j)}$ for all $i \in \mathcal{M}$ and $j \in H_{i}$.

If $\mathcal{E}_{\mathcal{M}}$ occurs, then there are $(\mathcal{P}, \mathcal{T})$ such that $\mathcal{E}_{\mathcal{M}}(\mathcal{P}, \mathcal{T})$ occurs. Using the union bound as in 33 , we get

$$
\mathrm{P}\left[\mathcal{E}_{\mathcal{M}}\right] \leq \sum_{\mathcal{P}, \mathcal{T}} \mathrm{P}\left[\mathcal{E}_{\mathcal{M}}(\mathcal{P}, \mathcal{T})\right] \leq\left[\left(\begin{array}{c}
k \\
1, l^{\prime}, l-l^{\prime}-1
\end{array}\right) t^{l^{\prime}} t^{l-l^{\prime}-1}\right]^{\mu} \max _{\mathcal{P}, \mathcal{T}} \mathrm{P}\left[\mathcal{E}_{\mathcal{M}}(\mathcal{P}, \mathcal{T})\right]
$$

Hence, we need to bound $\mathrm{P}\left[\mathcal{E}_{\mathcal{M}}(\mathcal{P}, \mathcal{T})\right]$ for any given $\mathcal{P}, \mathcal{T}$. To this end, let

$$
\begin{aligned}
\mathcal{I} & =\mathcal{I}(\mathcal{M}, \mathcal{P}, \mathcal{T})=\left\{(s, i, j): i \in \mathcal{M}, j \in P_{i}, s=t_{i}(j)\right\} \\
\mathcal{I}^{\prime} & =\mathcal{I}^{\prime}(\mathcal{M}, \mathcal{P}, \mathcal{T})=\left\{(s, i, j): i \in \mathcal{M}, j \in P_{i}^{\prime}, s=t_{i}(j)^{\prime}\right\}
\end{aligned}
$$

If $\mathcal{E}_{\mathcal{M}}(\mathcal{P}, \mathcal{T})$ occurs, then the positive literals of each clause $\boldsymbol{\Phi}_{i}$ are precisely $\boldsymbol{\Phi}_{i j}$ with $j \in P_{i}(i \in \mathcal{M})$. In addition, $\mathcal{H}_{s i j}^{\prime}=1$ for all $(s, i, j) \in \mathcal{I}$ and $\mathcal{H}_{s i j}^{\prime}=1$ for all $(s, i, j) \in \mathcal{I}^{\prime}$. Hence, by Lemmas 4.8 and 5.7

$$
\mathrm{P}\left[\mathcal{E}_{\mathcal{M}}(\mathcal{P}, \mathcal{T})\right] \leq 2^{-k \mu} \mathrm{E}\left[\prod_{(t, i, j) \in \mathcal{I}^{\prime}} \mathcal{H}_{t i j}^{\prime} \prod_{(t, i, j) \in \mathcal{I}} \mathcal{H}_{t i j} \mid \mathcal{F}_{0}\right] \leq\left[2^{-k}\left(\frac{3}{n-\theta-3 \theta^{\prime}}\right)^{l^{\prime}}\left(\frac{1}{n-\theta}\right)^{l-l^{\prime}-1}\right]^{\mu} \text { (37.) }
$$

Combining (36) and (37), we see that $\mathrm{P}\left[\mathcal{E}_{\mathcal{M}}\right] \leq \lambda^{\mu}$, where

$$
\begin{aligned}
\lambda & =2^{-k}\left(\begin{array}{c}
k \\
1, l^{\prime}, l-l^{\prime}-1
\end{array}\right)\left(\frac{3 t^{\prime}}{n-\theta-3 \theta^{\prime}}\right)^{l^{\prime}}\left(\frac{t}{n-\theta}\right)^{l-l^{\prime}-1} \\
& \leq k 2^{-k}\left(\begin{array}{c}
k-1 \\
l^{\prime}
\end{array}\right)\left(\frac{3 t^{\prime}}{n-\theta-3 \theta^{\prime}}\right)^{l^{\prime}} \cdot\left(\begin{array}{c}
k-l^{\prime}-1 \\
l-l^{\prime}-1
\end{array}\right)\left(\frac{t}{n-\theta}\right)^{l-l^{\prime}-1} \\
& \leq k 2^{-k} \cdot\left(\frac{6 k \theta^{\prime}}{n}\right)^{l^{\prime}}\left(\frac{\mathrm{e}\left(k-l^{\prime}-1\right) \theta}{\left(l-l^{\prime}-1\right) n}\right)^{l-l^{\prime}-1} .
\end{aligned}
$$

Invoking Lemma 2.2 we obtain $\mathrm{P}\left[\sum_{i=1}^{m} Y_{i}>2 \lambda m\right]=o\left(n^{-3}\right)$. Thus, we just need to show that $2 \lambda m<$ $\exp (-l) n$. Since $\theta / n \leq 4 k^{-1} \ln \omega$ and $\theta^{\prime} / n<k^{-2}$, in the case $l^{\prime} \geq l / 2$, (38) yields

$$
\lambda m \leq \omega n\left(4 \mathrm{e} \ln \omega \cdot \theta^{\prime} / n\right)^{l^{\prime} / 2} \leq \exp (-l) n / 2 .
$$

Furthermore, if $l^{\prime}<l / 2$, then we obtain from 38

$$
\lambda m \leq \omega n \exp \left(-2 l^{\prime}\right)(10 \mathrm{e} \ln \omega / l)^{l-l^{\prime}-1} \leq \exp (-l) n / 2 .
$$

Hence, in either case we obtain the desired bound. 
Proof of Lemma 5.3 Let $X\left(l, l^{\prime}, t, t^{\prime}\right)$ be the number of indices $i \in[m]$ such that $\boldsymbol{\Phi}_{i}$ satisfies a.-d. from Lemma 5.8 if $t \leq T$ and $t^{\prime} \leq T^{\prime}$, and set $X\left(l, l^{\prime}, t, t^{\prime}\right)=0$ if $t>T$ or $t^{\prime}>T^{\prime}$. Let $\mathcal{E}$ be the event that $T \leq \theta$ and $X\left(l, l^{\prime}, t, t^{\prime}\right) \leq \bar{B}\left(l, l^{\prime}, t\right)$ for all $2 \leq l \leq \sqrt{k}, 1 \leq l^{\prime} \leq l-1, t \leq \theta$, and $t^{\prime} \leq \theta^{\prime}$. Then by Corollary 4.4 and Lemma 5.8

$$
\mathrm{P}[\neg \mathcal{E}] \leq \mathrm{P}[T>\theta]+k \theta \theta^{\prime} \cdot o\left(n^{-3}\right)=o(1)
$$

Let $I_{l}$ be the number of indices $i \in U_{t^{\prime}} \backslash U_{T}$ and $\boldsymbol{\Phi}_{i}$ has precisely $l \leq \sqrt{k}$ positive literals. If $i$ has these properties, then $i$ satisfies the condition a. $-\mathrm{d}$. from Lemma 5.8 for $t=T$ and some $1 \leq l^{\prime}<l$. Therefore,

$$
\left|U_{t^{\prime}} \backslash U_{T}\right| \leq \sum_{l=1}^{k} I_{l}
$$

If the event $\mathcal{E}$ occurs, we have

$$
\begin{aligned}
\sum_{1 \leq l \leq \sqrt{k}} I_{l} & \leq \sum_{1 \leq l \leq \sqrt{k}} \sum_{l^{\prime}=1}^{l-1} X\left(l, l^{\prime}, T, t^{\prime}\right) \leq \sum_{l=1}^{k} \sum_{l^{\prime}=1}^{l-1} B\left(l, l^{\prime}, T\right) \\
& \leq 4 \omega n \sum_{l^{\prime}=1}^{k}\left(\frac{6 \theta^{\prime} k}{n}\right)^{l^{\prime}} \sum_{j=0}^{k-l^{\prime}-1}\left(\begin{array}{c}
k-l^{\prime}-1 \\
j
\end{array}\right)\left(\frac{T}{n}\right)^{j}(1-T / n)^{k-l^{\prime}-1-j} \\
& =4 \omega n \sum_{l^{\prime}=1}^{k}\left(\frac{6 \theta^{\prime} k}{n}\right)^{l^{\prime}} \leq 5 \omega n \cdot \frac{6 \theta^{\prime} k}{n} \leq n / k^{2} \quad\left[\text { because } \theta^{\prime}<n / k^{4}\right] .
\end{aligned}
$$

Furthermore, by Corollary 4.4 and Lemma 5.9 we have

$$
\sum_{\sqrt{k}<l \leq k} I_{l} \leq \sum_{\sqrt{k}<l \leq k} \exp (-l) n \leq n / k^{2} \quad \text { w.h.p. }
$$

Thus, the assertion follows from (39)- 42).

\subsection{Proof of Corollary 5.5}

As a preparation we need to estimate the number of clauses that have contain a huge number of literals from $Z_{t}$ for some $t \leq \theta$.

Lemma 5.10 Let $t \leq \theta$. With probability at least $1-o(1 / n)$ there are no more than $n \exp (-k)$ indices $i \in[m]$ such that $\left|\left\{j: k_{1}<j \leq k,\left|\Phi_{i j}\right| \in Z_{t}\right\}\right| \geq k / 4$.

Proof. For any $i \in[m], j \in[k]$, and $1 \leq s \leq \theta$ let

$$
\mathcal{Z}_{s i j}= \begin{cases}1 & \text { if }\left|\boldsymbol{\Phi}_{i j}\right|=z_{s}, \pi_{s-1}(i, j) \in\{-1,1\}, \text { and } s \leq T \\ 0 & \text { otherwise. }\end{cases}
$$

Then for any set $\mathcal{I} \subset[t] \times[m] \times\left([k] \backslash\left[k_{1}\right]\right)$ we have

$$
\mathrm{E}\left[\prod_{(s, i, j) \in \mathcal{I}} \mathcal{Z}_{s i j}\right] \leq(n-\theta)^{-|\mathcal{I}|} .
$$

To see this, let $\mathcal{I}_{s}=\{(i, j):(s, i, j) \in \mathcal{I}\}$ and set $\mathcal{Z}_{s}=\prod_{(i, j) \in \mathcal{I}_{s}} \mathcal{Z}_{s i j}$. Then for all $s \leq \theta$ the random variable $\mathcal{Z}_{s}$ is $\mathcal{F}_{s}$-measurable by Fact 4.1 Moreover, we claim that

$$
\mathrm{E}\left[\mathcal{Z}_{s} \mid \mathcal{F}_{s-1}\right] \leq(n-\theta)^{-\left|\mathcal{I}_{s}\right|}
$$


for any $s \leq \theta$. To prove this, consider any formula $\Phi$ such that $s \leq T[\Phi]$ and $\pi_{s-1}(i, j)[\Phi] \in\{-1,1\}$ for all $(i, j) \in \mathcal{I}_{s}$. Then by Proposition 4.2 in the probability distribution $\mathrm{P}\left[\cdot \mid \mathcal{F}_{s-1}\right](\Phi)$ the variables $\left(\boldsymbol{\Phi}_{i j}\right)_{(i, j) \in \mathcal{I}_{s}}$ are mutually independent and uniformly distributed over $V \backslash Z_{s-1}$. They are also independent of the variable $z_{s}$, because $j>k_{1}$ for all $(i, j) \in \mathcal{I}_{s}$ and the variable $z_{s}$ is determined by the first $k_{1}$ literals of some clause $\phi_{s}$ (cf. PI2). Therefore, for all $(i, j) \in \mathcal{I}_{s}$ the event $\boldsymbol{\Phi}_{i j}=z_{s}$ occurs with probability $1 /\left|V \backslash Z_{s-1}\right|$ independently. As $\left|Z_{s-1}\right|=s-1$, this shows (44), and (43) follows from Lemma 2.4 and (44).

Let $X_{i}=1$ if $t \leq T$ and there are at least $\kappa=\lceil k / 4\rceil$ indices $j \in[k] \backslash\left[k_{1}\right]$ such that $\left|\boldsymbol{\Phi}_{i j}\right| \in Z_{t}$, and set $X_{i}=0$ otherwise. Let $\mathcal{M} \subset[m]$ be a set of size $\mu=\left\lceil\ln ^{2} n\right\rceil$ and let $\mathcal{E}_{\mathcal{M}}$ be the event that $X_{i}=1$ for all $i \in \mathcal{M}$. Furthermore, let $P_{i} \subset[k] \backslash\left[k_{1}\right]$ be a set of size $\kappa-1$ for each $i \in \mathcal{M}$, and let $t_{i}: P_{i} \rightarrow[t]$ be a map. Let $\mathcal{P}=\left(P_{i}\right)_{i \in \mathcal{M}}$ and $\mathcal{T}=\left(t_{i}\right)_{i \in \mathcal{M}}$, and let $\mathcal{E}_{\mathcal{M}}(\mathcal{P}, \mathcal{T})$ be the event that $t \leq T$ and $\mathcal{Z}_{t_{i}(j) i j}=1$ for all $i \in \mathcal{M}$ and all $j \in P_{i}$. Let

$$
\mathcal{I}=\mathcal{I}_{\mathcal{M}}(\mathcal{P}, \mathcal{T})=\left\{\left(t_{i}(j), i, j\right): i \in \mathcal{M}, j \in P_{i}\right\} .
$$

Then (43) entails that for any $\mathcal{P}, \mathcal{T}$

$$
\mathrm{P}\left[\mathcal{E}_{\mathcal{M}}(\mathcal{P}, \mathcal{T})\right] \leq \mathrm{E}\left[\prod_{(s, i, j) \in \mathcal{I}} \mathcal{Z}_{s i j}\right] \leq(n-\theta)^{-|\mathcal{I}|} \leq(n-\theta)^{-\mu(\kappa-1)} .
$$

Moreover, if $\mathcal{E}_{\mathcal{M}}$ occurs, then there exist $\mathcal{P}, \mathcal{T}$ such that $\mathcal{E}_{\mathcal{M}}(\mathcal{P}, \mathcal{T})$ occurs. Hence, by the union bound

$$
\begin{aligned}
\mathrm{P}\left[\mathcal{E}_{\mathcal{M}}\right] & \leq \sum_{\mathcal{P}, \mathcal{T}} \mathrm{P}\left[\mathcal{E}_{\mathcal{M}}(\mathcal{P}, \mathcal{T})\right] \leq \lambda^{\mu} \quad \text { where } \\
\lambda & =\left(\begin{array}{c}
k-k_{1} \\
\kappa-1
\end{array}\right) t^{\kappa-1}(n-\theta)^{1-\kappa} \leq\left(\frac{\mathrm{e} k t}{(\kappa-1)(n-\theta)}\right)^{\kappa-1} \leq(12 \theta / n)^{\kappa-1} .
\end{aligned}
$$

Finally, Lemma2.2 implies that with probability $1-o\left(n^{-1}\right)$ we have

$$
\sum_{i=1}^{m} X_{i} \leq 2 m \lambda \leq n \cdot 2^{k}(12 \theta / n)^{\kappa-1} \leq n \exp (-k)
$$

as desired.

Proof of Corollary [5.5. We use a similar argument as in the proof of Corollary 4.7. Let

$$
\mathcal{U}_{t}^{\prime}=\left|\left\{x \in V \backslash\left(Z_{T} \cup Z_{t}^{\prime}\right): U_{t}^{\prime}(x)=0\right\}\right|,
$$

set $\alpha=\varepsilon / 3$, and define $0 / 1$ random variables $\mathcal{B}_{t}^{\prime}$ for $t \geq 1$ by letting $\mathcal{B}_{t}^{\prime}=1$ iff the following statements hold:
a. $T^{\prime} \geq t$.
b. $\mathcal{U}_{t-1}^{\prime} \geq n k^{\alpha-1}$.
c. There are less than $k / 4$ indices $k_{1}<j \leq k$ such that $\left|\Phi_{\psi_{t} j}\right| \in Z_{T}$.
d. There is $z \in Z_{t}^{\prime} \backslash Z_{t-1}^{\prime}$ such that $U_{t-1}^{\prime}(z)>0$.

This random variable is $\mathcal{F}_{t}^{\prime}$-measurable by Fact 5.1 Let $\delta=\exp \left(-k^{\alpha} / 6\right)$. We claim

$$
\mathrm{E}\left[\mathcal{B}_{t}^{\prime} \mid \mathcal{F}_{t-1}\right] \leq \delta \quad \text { for any } t \geq 1 .
$$

To see this, let $\Phi$ be a formula for which a.-c. hold. We condition on the event $\Phi \equiv_{t-1}^{\prime} \Phi$. Then at time $t$ the process PI1'-PI4' chooses $\psi_{t}$ such that $\boldsymbol{\Phi}_{\psi_{t}}$ contains less than three variables from $Z_{t-1}^{\prime}$. Since $\Phi$ satisfies c., there are less than $k / 4$ indices $j>k_{1}$ such that $\left|\boldsymbol{\Phi}_{\psi_{t} j}\right| \in Z_{T}$. Further, since $\boldsymbol{\Phi}_{\psi_{t}}$ is $\left(Z_{T}, Z_{t-1}^{\prime}\right)$-endangered, there is no $j$ such that $\pi_{t-1}^{\prime}\left(\psi_{t}, j\right)=1$. Consequently, there are at least $\frac{3}{4} k-$ 
$k_{1}-6 \geq k / 5$ indices $k_{1}<j \leq k-5$ such that $\pi_{t-1}^{\prime}\left(\psi_{t}, j\right)=-1$. Let $\mathcal{J}$ be the set of all these indices. Then Proposition 5.2 entails that in the distribution $\mathrm{P}\left[\cdot \mid \mathcal{F}_{t-1}^{\prime}\right](\Phi)$ the variables $\left(\left|\boldsymbol{\Phi}_{\psi_{t} j}\right|\right)_{j \in \mathcal{J}}$ are mutually independent and uniformly distributed over $V \backslash\left(Z_{T} \cup Z_{t-1}^{\prime}\right)$. Therefore, the number of indices $j \in \mathcal{J}$ such that $U_{t-1}^{\prime}\left(\left|\Phi_{\psi_{t} j}\right|\right)=0$ has a binomial distribution $\operatorname{Bin}\left(|\mathcal{J}|,\left|\mathcal{U}_{t-1}^{\prime}\right| /\left|V \backslash\left(Z_{T} \cup Z_{t-1}^{\prime}\right)\right|\right)$. If d. occurs, then there are less than three indices $j \in \mathcal{J}$ such that $U_{t-1}^{\prime}\left(\left|\Phi_{\psi_{t} j}\right|\right)=0$. Since $|\mathcal{J}| \geq k / 5$, b. and the Chernoff bound (1) yield

$$
\begin{aligned}
\mathrm{E}\left[\mathcal{B}_{t}^{\prime} \mid \mathcal{F}_{t-1}^{\prime}\right](\Phi) & \leq \mathrm{P}\left[\operatorname{Bin}\left(|\mathcal{J}|,\left|\mathcal{U}_{t-1}^{\prime}\right| /\left|V \backslash\left(Z_{T} \cup Z_{t-1}^{\prime}\right)\right|\right)<3\right] \\
& \leq \mathrm{P}\left[\operatorname{Bin}\left(\lceil k / 5\rceil, k^{\alpha-1}\right)<3\right] \leq \delta
\end{aligned}
$$

(provided that $k$ is sufficiently large). Thus, we have established (46).

Let $\mathcal{Y}^{\prime}=\left|\left\{t \in\left[\theta^{\prime}\right]: \mathcal{B}_{t}^{\prime}=1\right\}\right|$. We are going to show that

$$
\mathcal{Y}^{\prime} \leq 2 \theta^{\prime} \delta \quad \text { w.h.p. }
$$

To this end, letting $\mu=\lceil\ln n\rceil$, we will show that

$$
\mathrm{E}\left[\left(\mathcal{Y}^{\prime}\right)_{\mu}\right] \leq\left(\theta^{\prime} \delta\right)^{\mu} \quad \text { where }\left(\mathcal{Y}^{\prime}\right)_{\mu}=\prod_{j=0}^{\mu-1} \mathcal{Y}^{\prime}-j
$$

This implies (47). For if $\mathcal{Y}^{\prime}>2 \theta^{\prime} \delta$, then for large $n$ we have $\left(X^{\prime \prime}\right)_{\mu}>\left(2 \theta^{\prime} \delta-\mu\right)^{\mu} \geq\left(1.9 \cdot \theta^{\prime} \delta\right)^{\mu}$, whence Markov's inequality entails $\mathrm{P}\left[\mathcal{Y}^{\prime}>2 \theta^{\prime} \delta\right] \leq \mathrm{P}\left[\left(\mathcal{Y}^{\prime}\right)_{\mu}>\left(1.9 \theta^{\prime} \delta\right)^{\mu}\right] \leq 1.9^{-\mu}=o(1)$.

In order to establish (48), we define a random variable $\mathcal{Y}_{\mathcal{T}}^{\prime}$ for any tuple $\mathcal{T}=\left(t_{1}, \ldots, t_{\mu}\right)$ of mutually distinct integers $t_{1}, \ldots, t_{\mu} \in[\theta]^{\prime}$ by letting $\mathcal{Y}_{\mathcal{T}}^{\prime}=\prod_{i=1}^{\mu} \mathcal{B}_{t_{i}}^{\prime}$. Since $\left(\mathcal{Y}^{\prime}\right)_{\mu}$ equals the number of $\mu$-tuples $\mathcal{T}$ such that $\mathcal{Y}_{\mathcal{T}}^{\prime}=1$, we obtain

$$
\mathrm{E}\left[\left(\mathcal{Y}^{\prime}\right)_{\mu}\right] \leq \sum_{\mathcal{T}} \mathrm{E}\left[\mathcal{Y}_{\mathcal{T}}^{\prime}\right] \leq \theta^{\prime \mu} \max _{\mathcal{T}} \mathrm{E}\left[\mathcal{Y}_{\mathcal{T}}^{\prime}\right]
$$

To bound the last expression, we may assume that $\mathcal{T}$ is such that $t_{1}<\cdots<t_{\mu}$. As $\mathcal{B}_{t}^{\prime}$ is $\mathcal{F}_{t}^{\prime}$-measurable, we have for all $l \leq \mu$

$$
\mathrm{E}\left[\prod_{i=1}^{l} \mathcal{B}_{t_{i}}^{\prime}\right] \leq \mathrm{E}\left[\mathrm{E}\left[\prod_{i=1}^{l} \mathcal{B}_{t_{i}}^{\prime} \mid \mathcal{F}_{t_{l}-1}^{\prime}\right]\right]=\mathrm{E}\left[\prod_{i=1}^{l-1} \mathcal{B}_{t_{i}}^{\prime} \cdot \mathrm{E}\left[\mathcal{B}_{t_{l}}^{\prime} \mid \mathcal{F}_{t_{l}-1}^{\prime}\right] \stackrel{446}{\leq} \delta \cdot \mathrm{E}\left[\prod_{i=1}^{l-1} \mathcal{B}_{t_{i}}^{\prime}\right]\right.
$$

Proceeding inductively from $l=\mu$ down to $l=1$, we obtain $\mathrm{E}\left[\mathcal{Y}_{\mathcal{T}}^{\prime}\right] \leq \delta^{\mu}$, and thus (48) follows from (49).

To complete the proof, let $\mathcal{Y}^{\prime \prime}$ be the number of indices $i \in[m]$ such that $\left|\boldsymbol{\Phi}_{i j}\right| \in Z_{T}$ for at least $k / 4$ indices $k_{1}<j \leq k$. Combining Corollary 4.4 (which shows that $\left|Z_{T}\right|=T \leq \theta$ w.h.p.) with Lemma 5.10, we see that $\mathcal{Y}^{\prime \prime} \leq n \exp (-k) \leq \theta \delta$ w.h.p. As $|\mathcal{Y}| \leq \mathcal{Y}^{\prime}+\mathcal{Y}^{\prime \prime}$, the assertion thus follows from (47).

\subsection{Proof of Corollary 5.6}

Recall that a clause $\boldsymbol{\Phi}_{i}$ is $\left(Z_{T}, Z_{t}^{\prime}\right)$-endangered if for any $j$ such that the literal $\boldsymbol{\Phi}_{i j}$ is true under $\sigma_{Z_{T}}$ the underlying variable $\left|\Phi_{i j}\right|$ lies in $Z_{t}^{\prime}$. Let $\mathcal{Y}$ be the set from Corollary 5.5, and let $\mathcal{Z}=\bigcup_{s \in \mathcal{Y}} Z_{s} \backslash Z_{s-1}$. We claim that if $\boldsymbol{\Phi}_{i}$ is $\left(Z_{T}, Z_{t}^{\prime}\right)$-endangered, then one of the following statements is true:

a. There are two indices $1 \leq j_{1}<j_{2} \leq k$ such that $\left|\boldsymbol{\Phi}_{i j_{1}}\right|=\left|\boldsymbol{\Phi}_{i j_{2}}\right|$.

b. There are indices $i^{\prime} \neq i, j_{1} \neq j_{2}, j_{1}^{\prime} \neq j_{2}^{\prime}$ such that $\left|\boldsymbol{\Phi}_{i j_{1}}\right|=\left|\boldsymbol{\Phi}_{i^{\prime} j_{1}^{\prime}}\right|$ and $\left|\boldsymbol{\Phi}_{i j_{2}}\right|=\left|\boldsymbol{\Phi}_{i^{\prime} j_{2}^{\prime}}\right|$.

c. $\boldsymbol{\Phi}_{i}$ is unsatisfied under $\sigma_{Z_{T}}$.

d. $\boldsymbol{\Phi}_{i}$ contains more than $\kappa=\lfloor\sqrt{k}\rfloor$ positive literals, all of which lie in $Z_{t}^{\prime} \cup Z_{T}$.

e. $\boldsymbol{\Phi}_{i}$ has at most $\kappa$ positive literals, is satisfied under $\sigma_{Z_{T}}$, and contains a variable from $\mathcal{Z}$. 
To see this, assume that $\boldsymbol{\Phi}_{i}$ is $\left(Z_{T}, Z_{t}^{\prime}\right)$-endangered for some $t \leq T^{\prime}$ and a.-d. do not hold. Also observe that $\mathcal{Z} \supset Z_{T} \cap Z_{t}^{\prime}$ by construction (cf. PI2'); hence, if there is an index $j$ such that $\boldsymbol{\Phi}_{i j}=\bar{x}$ for some $x \in Z_{T}$, then $x \in \mathcal{Z}$, and thus e. holds. Thus, assume that no variable from $Z_{T}$ occurs negatively in $\boldsymbol{\Phi}_{i}$. Then $\boldsymbol{\Phi}_{i}$ contains $l \geq 1$ positive literals from $V \backslash Z_{T}$, and we may assume without loss of generality that these are just the first $l$ literals $\boldsymbol{\Phi}_{i 1}, \ldots, \boldsymbol{\Phi}_{i l}$. Furthermore, $\boldsymbol{\Phi}_{i 1}, \ldots, \boldsymbol{\Phi}_{i l} \in Z_{t}^{\prime}$. Hence, for each $1 \leq j \leq l$ there is $1 \leq t_{j} \leq t$ such that $\boldsymbol{\Phi}_{i j} \in Z_{t_{j}}^{\prime} \backslash Z_{t_{j}-1}^{\prime}$. Since $\boldsymbol{\Phi}_{i}$ satisfies neither a. nor b., the numbers $t_{1}, \ldots, t_{l}$ are mutually distinct. (For if, say, $t_{1}=t_{2}$, then either $\boldsymbol{\Phi}_{i 1}=\boldsymbol{\Phi}_{i 2}$, or $\boldsymbol{\Phi}_{i}$ and $\boldsymbol{\Phi}_{\psi_{t_{1}}}$ have at least two variables in common.) Thus, we may assume without loss of generality that $t_{1}<\cdots<t_{l}$. Then $i \in U_{t_{l}-1}^{\prime}$ by the construction in step PI3', and thus $\boldsymbol{\Phi}_{i l} \in \mathcal{Z}$. Hence, e. holds.

Let $X_{a}, \ldots, X_{e}$ be the numbers of indices $i \in[m]$ for which a.,.., e. above hold. W.h.p. $X_{a}+$ $X_{b}=O(\ln n)$ by Lemma 2.3 Furthermore, $X_{c} \leq \exp \left(-k^{\varepsilon / 8}\right) n$ w.h.p. by Proposition 3.2 Moreover, Lemmas 4.10 and 5.9 yield $X_{d} \leq 2 \exp (-\kappa / 2) n$ w.h.p. Finally, since $\mathcal{Y} \leq 3 \theta^{\prime} \exp \left(-k^{\varepsilon / 4}\right)$ w.h.p. by Corollary 5.5 and as $|\mathcal{Z}|=3|\mathcal{Y}|$, Lemma 2.6 shows that w.h.p.

$$
X_{e} \leq \sqrt{\theta^{\prime} \cdot 9 \exp \left(-k^{\varepsilon / 4}\right) n}<\theta^{\prime} / 2 .
$$

Combining these estimates, we obtain $X_{a}+\cdots+X_{e} \leq \theta^{\prime}$ w.h.p.

\section{Proof of Proposition 3.4}

As before, we let $0<\varepsilon<0.1$, and we assume that $k \geq k_{0}$ for a large enough $k_{0}=k_{0}(\varepsilon)$, and that $n>n_{0}$ for some large enough $n_{0}=n_{0}(\varepsilon, k)$. Furthermore, we let $m=\left\lfloor(1-\varepsilon) 2^{k} k^{-1} \ln k\right\rfloor, \omega=$ $(1-\varepsilon) \ln k$ and $k_{1}=\lceil k / 2\rceil$. We keep the notation introduced in Section 4.1] In particular, recall that $\theta=\left\lfloor 4 n k^{-1} \ln \omega\right\rfloor$.

In order to prove that the graph $G\left(\Phi, Z, Z^{\prime}\right)$ has a matching that covers all $\left(Z, Z^{\prime}\right)$-endangered clauses, we are going to apply the marriage theorem. Basically we are going to argue as follows. Let $Y \subset Z^{\prime}$ be a set of variables. Since $Z^{\prime}$ is "small" by Proposition 3.3. $Y$ is small, too. Furthermore, Phase 2 ensures that any $\left(Z, Z^{\prime}\right)$-endangered clause contains three variables from $Z^{\prime}$. To apply the marriage theorem, we thus need to show that w.h.p. for any $Y \subset Z^{\prime}$ the number of $\left(Z, Z^{\prime}\right)$-endangered clauses that contain only variables from $Y \cup\left(V \backslash Z^{\prime}\right)$ (i.e., the set of all $\left(Z, Z^{\prime}\right)$-endangered clauses whose neighborhood in $G\left(\boldsymbol{\Phi}, Z, Z^{\prime}\right)$ is a subset of $\left.Y\right)$ is at most $|Y|$.

To establish this, we will use a first moment argument (over sets $Y$ ). This argument does actually not take into account that $Y \subset Z^{\prime}$, but it works for any "small" set $Y \subset V$. Thus, let $Y \subset V$ be a set of size $y n$. We define a family $\left(y_{i j}\right)_{i \in[m], j \in[k]}$ of random variables by letting

$$
y_{i j}= \begin{cases}1 & \text { if }\left|\boldsymbol{\Phi}_{i j}\right| \in Y \\ 0 & \text { otherwise }\end{cases}
$$

Moreover, define for each integer $t \geq 0$ an equivalence relation $\equiv_{t}^{Y}$ on $\Omega_{k}(n, m)$ by letting $\Phi \equiv_{t}^{Y} \Phi^{\prime}$ iff $\pi_{s}[\Phi]=\pi_{s}\left[\Phi^{\prime}\right]$ for all $0 \leq s \leq t$ and $y_{i j}[\Phi]=y_{i j}\left[\Phi^{\prime}\right]$ for all $(i, j) \in[m] \times[k]$. This is a refinement of the equivalence relation $\equiv_{t}$ from Section 4.1. Let $\mathcal{F}_{t}^{Y}$ be the $\sigma$-algebra generated by the equivalence classes of $\equiv_{t}^{Y}$. Then the family $\left(\mathcal{F}_{t}^{Y}\right)_{t \geq 0}$ is a filtration. Since $\mathcal{F}_{t}^{Y}$ contains the $\sigma$-algebra $\mathcal{F}_{t}$ from Section 4.1 all random variables that are $\mathcal{F}_{t}$-measurable are $\mathcal{F}_{t}^{Y}$-measurable as well.

Proposition 6.1 Let $\mathcal{E}_{t}^{Y}$ be the set of all pairs $(i, j)$ such that $\pi_{t}(i, j) \in\{1,-1\}$ and $y_{i j}=0$. The conditional joint distribution of the variables $\left(\left|\boldsymbol{\Phi}_{i j}\right|\right)_{(i, j) \in \mathcal{E}_{t}^{Y}}$ given $\mathcal{F}_{t}^{Y}$ is uniform over $\left(V \backslash\left(Z_{t} \cup Y\right)\right)^{\mathcal{E}_{t}^{Y}}$.

Proof. Let $[\Phi]_{t}^{Y}$ be the $\equiv_{t}^{Y}$-class of a formula $\Phi$. Then $\mathrm{P}_{\Phi}=\mathrm{P}\left[\cdot \mid \mathcal{F}_{t}^{Y}\right](\Phi)$ is just the uniform distribution over $[\Phi]_{t}^{Y}$. Let $\mathcal{D}_{t}^{Y}(\Phi)$ be the set of all pairs $(i, j) \in[m] \times k$ such that $\left|\Phi_{i j}\right| \in Y$ and $\pi_{t}(i, j)[\Phi] \in$ $\{-1,1\}$. We will actually prove the following stronger statement: with respect to the measure $\mathrm{P}_{\Phi}$ the joint distribution of the variables $\left(\left|\boldsymbol{\Phi}_{i j}\right|\right)_{(i, j) \in \mathcal{E}_{t}^{Y} \cup \mathcal{D}_{t}^{Y}}$ is uniform over $\left(V \backslash\left(Z_{t} \cup Y\right)\right)^{\mathcal{E}_{t}^{Y}} \times\left(Y \backslash Z_{t}\right)^{\mathcal{D}_{t}}$. 
To show this, we use a similar argument as in the proof of Proposition 4.2 For any two maps $f$ : $\mathcal{E}_{t}^{Y}(\Phi) \rightarrow V \backslash\left(Y \cup Z_{t}(\Phi)\right)$ and $g: \mathcal{D}_{t}^{Y}(\Phi) \rightarrow Y \backslash Z_{t}(\Phi)$ we define a formula

$$
\left(\Phi_{f, g}\right)_{i j}=\left\{\begin{array}{cl}
\overline{f(i, j)} & \text { if }(i, j) \in \mathcal{E}_{t}(\Phi) \text { and } \pi_{0}(i, j)=-1 \\
\frac{f(i, j)}{g(i, j)} & \text { if }(i, j) \in \mathcal{E}_{t}(\Phi) \text { and } \pi_{0}(i, j)=1 \\
g(i, j) & \text { if }(i, j) \in \mathcal{D}_{t}(\Phi) \text { and } \pi_{0}(i, j)=-1 \\
\Phi_{i j} & \text { otherwise. }
\end{array}\right.
$$

Then $\Phi_{f, g} \equiv_{t}^{Y} \Phi$. Therefore, the map

$$
\left(V \backslash\left(Z_{t} \cup Y\right)\right)^{\mathcal{E}_{t}^{Y}} \times\left(Y \backslash Z_{t}\right)^{\mathcal{D}_{t}^{Y}} \rightarrow[\Phi]_{t},(f, g) \mapsto \Phi_{f, g}
$$

is bijection.

For any $t \geq 1, i \in[m], j \in[k]$ we define a $0 / 1$ random variable $\mathcal{H}_{t i j}^{Y}$ by letting $\mathcal{H}_{t i j}^{Y}=1$ if $y_{i j}=0$, $t \leq T, \pi_{t-1}(i, j)=1$ and $\pi_{t}(i, j)=z_{t}$.

Lemma 6.2 For any set $\mathcal{I} \subset[\theta] \times[m] \times[k]$ we have $\mathrm{E}\left[\prod_{(t, i, j) \in \mathcal{I}} \mathcal{H}_{t i j}^{Y} \mid \mathcal{F}_{0}^{Y}\right] \leq(n-\theta)^{-|\mathcal{I}|}$.

Proof. Due to Proposition 6.1 the proof of Lemma 4.8 carries over directly.

For a given set $Y$ we would like to bound the number of $i \in[m]$ such that $\boldsymbol{\Phi}_{i}$ contains at least three variables from $Y$ and $\boldsymbol{\Phi}_{i}$ has no positive literal in $V \backslash\left(Y \cup Z_{T}\right)$. If for any "small" set $Y$ the number of such clauses is less than $|Y|$, then we can apply this result to $Y=Z^{\prime}$ and use the marriage theorem to show that $G\left(\Phi, Z, Z^{\prime}\right)$ has the desired matching. We proceed in several steps.

Lemma 6.3 Let $t \leq \theta$, let $\mathcal{M} \subset[m]$ be a set of size $\mu$, and let $L, \Lambda$ be maps that assign a subset of $[k]$ to each $i \in \mathcal{M}$ such that

$$
L(i) \cap \Lambda(i)=\emptyset \text { and }|\Lambda(i)| \geq 3 \text { for all } i \in \mathcal{M} \text {. }
$$

Let $\mathcal{E}(Y, t, \mathcal{M}, L, \Lambda)$ be the event that the following statements are true for all $i \in \mathcal{M}$ :

a. $\left|\boldsymbol{\Phi}_{i j}\right| \in Y$ for all $j \in \Lambda(i)$.

b. $\boldsymbol{\Phi}_{i j}$ is a negative literal for all $j \in[k] \backslash(L(i) \cup \Lambda(i))$.

c. $\boldsymbol{\Phi}_{i j} \in Z_{t} \backslash Y$ for all $j \in L(i)$.

Let $l=\sum_{i \in \mathcal{M}}|L(i)|$ and $\lambda=\sum_{i \in \mathcal{M}}|\Lambda(i)|$. Then $\mathrm{P}[\mathcal{E}(Y, t, \mathcal{M}, L, \Lambda)] \leq 2^{-k \mu}(2 t / n)^{l}(2 y)^{\lambda}$.

Proof. Let $\mathcal{E}=\mathcal{E}(Y, t, \mathcal{M}, L, \Lambda)$. Let $t_{i}$ be a map $L(i) \rightarrow[t]$ for each $i \in \mathcal{M}$, let $\mathcal{T}=\left(t_{i}\right)_{i \in \mathcal{M}}$, and let $\mathcal{E}(\mathcal{T})$ be the event that a. and b. hold and $\Phi_{i j}=z_{t_{i}(j)}$ for all $i \in \mathcal{M}$ and $j \in L(i)$. If $\mathcal{E}$ occurs, then there is $\mathcal{T}$ such that $\mathcal{E}(\mathcal{T})$ occurs. Hence, by the union bound

$$
\mathrm{P}[\mathcal{E}] \leq \sum_{\mathcal{T}} \mathrm{P}[\mathcal{E}(\mathcal{T})] \leq t^{l} \max _{\mathcal{T}} \mathrm{P}[\mathcal{E}(\mathcal{T})]
$$

To bound the last term fix any $\mathcal{T}$. Let $\mathcal{I}=\left\{(s, i, j): i \in \mathcal{M}, j \in L(i), s=t_{i}(j)\right\}$. If $\mathcal{E}(\mathcal{T})$ occurs, then $\mathcal{H}_{s i j}^{Y}=1$ for all $(s, i, j) \in \mathcal{I}$. Therefore, by Lemma6.2

$$
\mathrm{P}\left[\mathcal{E}(\mathcal{T}) \mid \mathcal{F}_{0}^{Y}\right] \leq \mathrm{E}\left[\prod_{(s, i, j) \in \mathcal{I}} \mathcal{H}_{s i j}^{Y} \mid \mathcal{F}_{0}^{Y}\right] \leq(n-\theta)^{-|\mathcal{I}|}=(n-\theta)^{-l}
$$

Furthermore, the event that a. and b. hold for all $i \in \mathcal{M}$ is $\mathcal{F}_{0}^{Y}$-measurable. Since the literals $\boldsymbol{\Phi}_{i j}$ are chosen independently, we have

$$
\mathrm{P}[\mathrm{a} . \text { and b. hold for all } i \in \mathcal{M}] \leq y^{\lambda} 2^{\lambda-k \mu}=(2 y)^{\lambda} 2^{-k \mu}
$$


Combining (52) and (53), we obtain $\mathrm{P}[\mathcal{E}(\mathcal{T})] \leq 2^{-k \mu}(n-\theta)^{-l}(2 y)^{\lambda}$. Finally, plugging this bound into (51), we get

$$
\mathrm{P}[\mathcal{E}] \leq 2^{-k \mu}\left(\frac{t}{n-\theta}\right)^{l}(2 y)^{\lambda} \leq 2^{-k \mu}\left(\frac{2 t}{n}\right)^{l}(2 y)^{\lambda}
$$

as desired.

Corollary 6.4 Let $t \leq \theta$, and let $\mathcal{M} \subset V$ have size $|\mathcal{M}|=\mu$. Let $l, \lambda$ be integers such that $\lambda \geq 3 \mu$. Let $\mathcal{E}(Y, t, \mathcal{M}, l, \lambda)$ be the event that there exist maps $L, \Lambda$ that satisfy (50) such that $l=\sum_{i \in \mathcal{M}}|L(i)|$, $\lambda=\sum_{i \in \mathcal{M}}|\Lambda(i)|$, and the event $\mathcal{E}(Y, t, \mathcal{M}, L, \Lambda)$ occurs. Then

$$
\mathrm{P}[\mathcal{E}(Y, t, \mathcal{M}, l, \lambda)] \leq 2^{-l-k \mu}\left(2 k^{2} y\right)^{\lambda} .
$$

Proof. Given $l, \lambda$ there are at most $\left(\begin{array}{l}k \mu \\ l, \lambda\end{array}\right)$ ways to choose the maps $L, \Lambda$ (because the clauses in $\mathcal{M}$ contain a total number of $k \mu$ literals). Therefore, by Lemma 6.3 and the union bound

$$
\begin{aligned}
2^{k \mu} \mathrm{P}[\mathcal{E}(Y, t, \mathcal{M}, l, \lambda)] & \leq\left(\begin{array}{c}
k \mu \\
l, \lambda
\end{array}\right)(2 t / n)^{l}(2 y)^{\lambda} \leq 2^{-l}\left(\frac{4 \mathrm{e} \theta k \mu}{\ln }\right)^{l}\left(\frac{2 \mathrm{e} k \mu y}{\lambda}\right)^{\lambda} \leq 2^{-l}\left(\frac{50 \mu \ln \omega}{l}\right)^{l}(2 k y)^{\lambda} \\
& =2^{-l}(2 k y)^{\lambda} \cdot \omega^{-50 \mu \cdot \alpha \ln \alpha}, \quad \text { where } \alpha=\frac{l}{50 \mu \ln \omega}
\end{aligned}
$$

Since $-\alpha \ln \alpha \leq 1 / 2$, we obtain $\omega^{-50 \mu \cdot \alpha \ln \alpha} \leq \omega^{-25 \mu} \leq(\ln k)^{25 \mu} \leq k^{\lambda}$. Plugging this last estimate into (54) yields the desired bound.

Corollary 6.5 Let $t \leq \theta$ and let $\mathcal{E}(t)$ be the event that there are sets $Y \subset V, \mathcal{M} \subset[m]$ of size $3 \leq$ $|Y|=|\mathcal{M}|=\mu \leq n k^{-12}$ and integers $l \geq 0, \lambda \geq 3 \mu$ such that the event $\mathcal{E}(Y, t, \mathcal{M}, l, \lambda)$ occurs. Then $\mathrm{P}[\mathcal{E}(t)]=o(1 / n)$.

Proof. Let us fix an integer $1 \leq \mu \leq n k^{-12}$ and let $\mathcal{E}(t, \mu)$ be the event that there exist sets $Y, \mathcal{M}$ of the given size $\mu=y n$ and numbers $l, \lambda$ such that $\mathcal{E}(Y, t, \mathcal{M}, l, \lambda)$ occurs. Then the union bound and Corollary 6.4 yield

$$
\begin{aligned}
\mathrm{P}[\mathcal{E}(t, \mu)] & \leq \sum_{\lambda \geq 3 \mu} \sum_{Y, \mathcal{M}:|Y|=|\mathcal{M}|=\mu} \sum_{l \geq 0} \mathrm{P}[\mathcal{E}(Y, t, \mathcal{M}, l, \lambda)] \leq\left(\begin{array}{c}
n \\
\mu
\end{array}\right)\left(\begin{array}{c}
m \\
\mu
\end{array}\right) 2^{2-k \mu}\left(2 k^{2} y\right)^{3 \mu} \\
& \leq\left(\frac{\mathrm{e}^{2} 2^{k} \ln \omega}{k y^{2}}\right)^{\mu} \cdot 2^{2-k \mu}\left(2 k^{2} y\right)^{3 \mu} \leq 4\left[y k^{6}\right]^{\mu} \leq y^{-\mu / 2} .
\end{aligned}
$$

Summing over $3 \leq \mu \leq n k^{-12}$, we obtain $\mathrm{P}[\mathcal{E}(t)] \leq \sum_{\mu} \mathrm{P}[\mathcal{E}(t, \mu)]=O\left(n^{-3 / 2}\right)$.

Proof of Proposition 3.4 Assume that the graph $G\left(\Phi, Z, Z^{\prime}\right)$ does not have a matching that covers all $\left(Z, Z^{\prime}\right)$-endangered clauses. Then by the marriage theorem there are a set $Y \subset Z^{\prime}$ and a set $\mathcal{M}$ of $\left(Z, Z^{\prime}\right)$ endangered clauses such that $|\mathcal{M}|=|Y|>0$ and all neighbors of indices $i \in \mathcal{M}$ in the graph $G\left(\Phi, Z, Z^{\prime}\right)$ lie in $Y$. Indeed, as each $\left(Z, Z^{\prime}\right)$-endangered clause contains at least three variables from $Z^{\prime}$, we have $|Y| \geq 3$. Therefore, for each clause $i \in \mathcal{M}$ the following three statements are true:

a. There is a set $\Lambda(i) \subset[k]$ of size at least 3 such that $\left|\boldsymbol{\Phi}_{i j}\right| \in Y$ for all $j \in \Lambda(i)$.

b. There is a (possibly empty) set $L(i) \subset[k] \backslash \Lambda(i)$ such that $\boldsymbol{\Phi}_{i j} \in Z$ for all $j \in L(i)$.

c. For all $j \in[k] \backslash(L(i) \cup \Lambda(i))$ the literal $\boldsymbol{\Phi}_{i j}$ is negative.

As a consequence, at least one of the following events occurs:

1. $T>\theta=\left\lfloor 4 k^{-1} \ln \omega\right\rfloor$.

2. $\left|Z^{\prime}\right|>n k^{-12}$.

3. There is $t \leq \theta$ such that $\mathcal{E}(t)$ occurs.

The probability of the first event is $o(1)$ by Proposition 3.2 , the second event has probability $o(1)$ by Proposition 3.3 and the probability of the third event is $\theta \cdot o\left(n^{-1}\right)=o(1)$ by Corollary 6.5 


\section{References}

[1] D. Achlioptas, P. Beame, M. Molloy: Exponential bounds for DPLL below the satisfiability threshold. Proc. 15th SODA (2004) 139-140.

[2] D. Achlioptas, A. Coja-Oghlan: Algorithmic barriers from phase transitions. Proc. 49th FOCS (2008) 793-802.

[3] D. Achlioptas, C. Moore: Random $k$-SAT: two moments suffice to cross a sharp threshold. SIAM Journal on Computing 36 (2006) 740-762.

[4] D. Achlioptas, Y. Peres: The threshold for random $k$-SAT is $2^{k} \ln 2-O(k)$. Journal of the AMS 17 (2004) 947-973.

[5] J. Ardelius, L. Zdeborova: Exhaustive enumeration unveils clustering and freezing in random 3-SAT. Phys. Rev. E 78 (2008) 040101(R).

[6] A. Braunstein, M. Mézard, R. Zecchina: Survey propagation: an algorithm for satisfiability. Random Structures and Algorithms 27 (2005) 201-226.

[7] M.-T. Chao, J. Franco: Probabilistic analysis of a generalization of the unit-clause literal selection heuristic for the $k$-satisfiability problem. Inform. Sci. 51 (1990) 289-314.

[8] V. Chvátal, B. Reed: Mick gets some (the odds are on his side). Proc. 33th FOCS (1992) 620-627.

[9] A. Coja-Oghlan, U. Feige, A. Frieze, M. Krivelevich, D. Vilenchik: On smoothed $k$-CNF formulas and the Walksat algorithm. Proc. 20th SODA (2009) 451-460.

[10] M. Davis, G. Longemann, D. Loveland: A machine program for theorem proving. Communications of the ACM 5 (1962) 394-397.

[11] O. Dubois, Y. Boufkhad, J. Mandler: Typical random 3-SAT formulae and the satisability threshold. Proc. 11th SODA (2000) 126-127.

[12] R. Durrett: Probability and examples. 3rd edition (2005).

[13] A. Flaxman: Algorithms for random 3-SAT. To appear.

[14] E. Friedgut: Sharp thresholds of graph properties, and the $k$-SAT problem. Journal of the AMS 12 (1999) 1017-1054.

[15] A. Frieze, S. Suen: Analysis of two simple heuristics on a random instance of $k$-SAT. Journal of Algorithms 20 (1996) 312-355.

[16] E. Goldberg, Y. Novikov: BerkMin: a fast and robust sat-solver. Discrete Applied Mathematics 155 (2007) 1549-1561.

[17] M. Hajiaghayi, G. Sorkin: The satisfiability threshold of random 3-SAT is at least 3.52. IBM Research Report RC22942 (2003).

[18] S. Janson, T. Łuczak, A. Ruciński: Random Graphs, Wiley 2000.

[19] J.H. Kim: Poisson cloning model for random graph. Preprint (2006).

[20] F. Krzakala, A. Montanari, F. Ricci-Tersenghi, G. Semerjian, L. Zdeborova: Gibbs states and the set of solutions of random constraint satisfaction problems. Proc. National Academy of Sciences 104 (2007) 10318-10323.

[21] A. Montanari, F. Ricci-Tersenghi, G. Semerjian: Solving constraint satisfaction problems through Belief Propagation-guided decimation. Proc. 45th Allerton (2007).

[22] G. Semerjian, R. Monasson: A study of pure random walk on random satisfiability problems with "physical" methods. Proc. 6th SAT (2003) 120-134. 